\title{
Catechol, a Natural Allelochemical Thwarts Virulent Dimorphism in Candida Albicans and Potentiates the Antifungal Efficacy of Azoles and Polyenes.
}

Jothi Ravi

Alagappa University

Sangavi Ravichellam

Alagappa University

Kumar Ponnuchamy

Alagappa University

Karutha Pandian Shunmugiah

Alagappa University

Gowrishankar Shanmugaraj ( $\square$ gowrishankar.alu@gmail.com )

Alagappa University

Research Article

Keywords: Candida albicans, catechol, antivirulence agent, antihyphal efficacy, antivirulence, antifungals

Posted Date: July 9th, 2021

DOI: https://doi.org/10.21203/rs.3.rs-668716/v1

License: (a) (1) This work is licensed under a Creative Commons Attribution 4.0 International License. Read Full License 


\section{Abstract}

The present study was deliberately focused to explore the antivirulence efficacy of a plant allelochemical catechol against Candida albicans, and attempts were made to elucidate the underlying mechanisms as well. Catechol at its sub-MIC concentrations ( 2 to $256 \mu \mathrm{g} / \mathrm{mL}$ ) exhibited a dose dependent biofilm as well as hyphal inhibitory efficacies, which were ascertained through both light and fluorescence microscopic analyses. Further, sub-MICs of catechol displayed remarkable antivirulence efficacy, as it substantially inhibited C. albicans' virulence enzymes i.e. secreted hydrolases. Notably, FTIR analysis divulged the potency of catechol in effective loosening of $C$. albicans' exopolymeric matrix, which was further reinforced using EPS quantification assay. Although, catechol at BIC $(256 \mu \mathrm{g} / \mathrm{mL})$ did not disrupt the mature biofilms of $C$. albicans, their initial adherence was significantly impeded by reducing their hydrophobic nature. Besides, FTIR analysis also unveiled the ability of catechol in enhancing the production of farnesol -a metabolite of $C$. albicans, whose accumulation naturally blocks yeast-hyphal transition. The qPCR data showed significant down-regulation of candidate genes viz., RAS1, HWP1 and ALS3 which are responsible for the regulation of Ras-cAMP-PKA pathway -the pathway that contribute for $C$. albicans' pathogenesis. Interestingly, the up-regulation of TUP1 (a gene responsible for farnesol-mediated hyphal inhibition) during catechol exposure strengthen the speculation of catechol triggered

farnesol-mediated hyphal inhibition. Furthermore, catechol profusely enhanced the fungicidal efficacy of certain known antifungal agent's viz., azoles (ketoconazole and miconazole) and polyenes (amphotericin-B and nystatin).

\section{Introduction}

Over the past few decades, the prevalence of invasive fungal infections caused by Candida species has been persistently increasing especially in immunosuppressed individuals ${ }^{1,2}$. Globally, most individuals encounter curable superficial fungal infections at least once in their lifetime. However, millions of people suffer from lifethreatening invasive fungal infection, which is much harder to diagnose and treat ${ }^{3}$. The global incidence of candidemia has been estimated at 6.0 to 13.3 cases per 100000 population with an associated mortality rate of $50 \%$, which is predicted to be even higher than the death rate caused by tuberculosis or malaria ${ }^{4}$. It has been reported that about $80 \%$ of nosocomial fungal infections are caused by Candida species. Amongst, Candida albicans, Candida tropicalis and Candida glabrata were found to be the major players ${ }^{5}$. In the midst, $C$. albicans is by far the most prevalent etiological agent of invasive candidiasis and are often ranked top in the list of most common fungal pathogens, causing severe disseminated infections in humans ${ }^{6}$. In United States, candidemia account for nearly $9 \%$ of nosocomial infections, of which $40-70 \%$ have been caused by C. albicans ${ }^{7}$. C. albicans being a diploid microorganism, it exists either in the form of yeast or hyphae, and reside as commensal in half of the healthy human population where it is controlled by the host and microbial interactions ${ }^{8}$. On the other hand, they can be able to cause irreversible pathogenic effect ranging from superficial to life-threatening systemic infections through its intrinsic yeast-to-hyphal transforming ability 9 .

C. albicans establish infection through four stages viz., colonization, superficial, deep-seated and systemic ${ }^{10}$. In the colonization stage, the growth of $C$. albicans is balanced by the host immune system and microflora. Nevertheless, the other three stages occur only under opportunistic circumstances promoting the overgrowth of C. albicans, which culminate in microflora imbalance and weakened immunity ${ }^{11}$. Yeast form of $C$. albicans plays an inevitable role in the process of colonization as it assists in both tissue penetration and host immune 
evasion. However, the hyphal form is indispensable for the pathogenesis of $C$. albicans, as it encodes several virulence genes such as secretion of adhesin, hydrolases and protease leading to host tissue damage ${ }^{2}$. Hence, the yeast to hyphal transition has been considered as a crucial virulence determinant of $C$. albicans. A mounting body of mutation studies reinforced that $C$. albicans mutants lacking yeast to hyphal transition displayed avirulent properties in vivo mouse models ${ }^{12,13}$. Apart from morphological plasticity, myriad of factors including metabolic adaptability (ability to survive in a broad range of $\mathrm{pH}$ ), biofilm formation, contact sensing and thigmotropism contributes to the pathogenesis of . albicans $^{14}$. At present, the therapeutic choices of antifungal agents are mostly restricted to azoles, polyenes and echinocandins. Unfortunately, these treatment choices have become unsatisfactory owing to the increased development of resistance, selective pressure, nonavailability of conventional antifungal for systemic administration and adverse effects at higher concentrations ${ }^{15}$. Thus, there is an urgent need to develop new agents with low toxicity, broad spectrum of activity and a new mode of action against drug-resistant $C$. albicans. In order to address the above-mentioned scientific issues, therapies that specifically target virulence rather than harming the fungal metabolism have been proved to be even more promising. One such remarkable approach is the antivirulence therapy, wherein biomolecules with proficiency to exclusively hamper the virulence traits of pathogens have been envisaged with the certainty that it would nullify the phenomenon of resistance by counteracting selection pressure ${ }^{16}$. In this milieu, plant-derived molecules have been attaining a great deal of attention to be an ideal antivirulence agent due to their drug-like properties ${ }^{17}$. Lately, allelochemicals have been emphasized as a natural antivirulent owing to their short half-life i.e. rapid biodegradability ${ }^{18,19}$.

Catechol $\left(\mathrm{C}_{6} \mathrm{H}_{6} \mathrm{O}_{2}\right)$, a benzenediol comprises of benzene ring at center coupled with two hydroxy substituents. It is one of the natural allelochemicals synthesized by plants especially in onions through the shikimate pathway ${ }^{20,21}$. Hitherto, a plethora of investigations have documented the multifaceted pharmaceutical properties of catechol viz., broad-spectrum antibacterial ${ }^{19}$, anticariogenic ${ }^{21}$ and antifungal ${ }^{18}$ efficacies. In addition, some reports have been revealed that catechol act as a stimulator for the root hair elongation in rice through reactive oxygen species mediated redox signaling ${ }^{19}$. Given the prominence of myriad therapeutic potentials of catechol, the compounds with catechol groups have been deployed in medical applications chiefly due to low cytotoxicity rendered by the holistic structure ${ }^{22,23}$. In fact, catechol moieties (such as dopamine and norepinephrine) found in the human body serves as essential regulators of central nervous system ${ }^{24}$. Thus, they are considered to be ecologically and toxicologically safer than the synthesized molecules. Nevertheless, its antivirulence propensity to impede the formation of biofilm and production of virulence in pathogenic fungi, especially $C$. albicans has not been explored till date. Therefore, the present study was consciously aimed to explore and delineate the inhibitory efficacy of catechol against biofilm assemblage, hyphal transition, and secreted virulence in C. albicans.

\section{Materials And Methods}

\subsection{Preparation and UV- spectral analysis of active compound}

The active compound catechol (purity $\geq 99 \%$ CAS Number: $120-80-9$ ) was purchased from Sigma-Aldrich. The stock solution was prepared as $100 \mathrm{mg} / \mathrm{mL}$ using ethanol and stored at $4^{\circ} \mathrm{C}$ for further use. To determine the influence of solvent (ethanol) on C. albicans biofilm and growth, the medium with inoculum and ethanol was set 
as a vehicle control. Furthermore, the catechol was scanned from 200 to $800 \mathrm{~nm}$ using UV- spectrophotometer to obtain the absorbance spectra of a catechol

\subsection{Strain and culture conditions}

The test organism C. albicans ATCC 10231 used in this study was purchased from HiMedia, India. The strain was maintained in sabouraud dextrose agar (SDA) plates and routinely cultured in yeast extract peptone dextrose (YEPD) broth at $37^{\circ} \mathrm{C}$. To perform all in vitro assays, $1 \%$ of $3 \mathrm{~h}$ culture with 0.1 optical density (OD) (1 $\times 10^{6} \mathrm{CFU} / \mathrm{mL}$ ) was used to inoculate in YEPD broth. Both biofilm and hyphal assays were performed in spider medium (consisting mannitol $1 \%, \mathrm{~K}_{2} \mathrm{HPO}_{4} 0.2 \%$ and nutrient broth $1 \%$ ) to allow hyphal elongation. Glucosephosphate-proline growth-medium (GPP) was used for the extraction of quorum sensing molecule (farnesol) from C. albicans.

\subsection{Evaluation of inhibitory effect of catechol on $C$. albicans planktonic growth}

To ensure whether the catechol at varied range of concentrations influence the growth of $C$. albicans planktonic cells, broth microdilution assay was performed following clinical \& laboratory standards institute (CLSI) 2008 standard guidelines with small alterations ${ }^{25}$. C. albicans $\left(1 \times 10^{6} \mathrm{CFU} / \mathrm{mL}\right)$ was dispensed into 24 -well microtiter plates (MTPs) containing YEPD broth supplemented with and without catechol at various concentrations (0$1024 \mu \mathrm{g} / \mathrm{mL}$ ) to a final volume of $1 \mathrm{~mL} /$ well. The YEPD medium supplemented with $C$. albicans devoid of catechol was served as a control. After incubation at $37^{\circ} \mathrm{C}$ for $24 \mathrm{~h}$, MIC was determined by measuring the OD at $600 \mathrm{~nm}$ using spectrophotometer (Spectra Max 3, Molecular Devices, United States). The MIC was defined as the lowest concentration of catechol that showed visible growth inhibition in YEPD broth compared to untreated control. All experiments were carried out in triplicate.

\subsection{Alamar blue assay}

Furthermore, the alamar blue assay was used to evaluate the metabolic viability of catechol treated samples as previously described by Repp et al., (2007) with minor modification ${ }^{26}$. Briefly, C. albicans cells were treated with catechol for $24 \mathrm{~h}$ at $37^{\circ} \mathrm{C}$. The $C$. albicans without catechol treatment was acted as control. After incubation, the culture suspension was centrifuged at 8,000 rpm for $10 \mathrm{~min}$ and the obtained pellet resuspended with sterile phosphate-buffered saline (PBS). Alamar blue $(100 \mu \mathrm{g} / \mathrm{mL})$ (Sigma Aldrich) was added to each cell pellets that were suspended in PBS and incubated at dark for 4-8 h. Subsequently, the fluorescent intensity of excitation $(560 \mathrm{~nm})$ and emission (590 nm) wavelengths were measured spectroscopically.

\subsection{Investigation of inhibitory effect of catechol on C. albicans biofilm}

In order to examine the antibiofilm efficacy of catechol at its sub-MIC against $C$. albicans biofilm, crystal violet staining method was performed according to the protocol prescribed by Prasath et al., (2019) with required modifications ${ }^{27}$. Briefly, overnight culture of $C$. albicans was inoculated into 24-well MTPs having $1 \mathrm{~mL}$ spider medium supplemented with catechol at various concentrations $(0-1024 \mu \mathrm{g} / \mathrm{mL})$, and incubated at $37^{\circ} \mathrm{C}$ for 48 h without shaking to allow the formation of biofilm. The spider medium supplemented with $C$. albicans devoid of catechol was considered as control. After incubation, the spent medium was transferred to fresh 24- well MTPs and read at $600 \mathrm{~nm}$ to check the impact of catechol on C. albicans planktonic cells. Further, the non- 
adherent planktonic cells on MTP were removed by washing with sterile PBS. Then, the sessile cells on the bottom of MTP were stained for 15 min with $0.4 \%$ crystal violet (HiMedia, India). Any excess stain was removed by washing with sterile water. After 15 min of destaining with $15 \%$ glacial acetic acid, the amount of crystal violet bound to the biofilm cells was quantified spectophotometrically at $570 \mathrm{~nm}$. The percentage of biofilm inhibition was determined using the following formula.

The relative biofilm inhibition: \% of biofilm inhibition $=[($ Control OD570 nm - Treated OD570 nm)/ Control OD570 nm] $\times 100$.

Biofilm Inhibitory concentration (BIC) was defined as the minimal concentration of catechol that brings about 90 $\%$ of biofilm inhibition without affecting cellular viability.

\subsection{Microscopic visualization of $C$. albicans biofilm}

In order to further ascertain the antibiofilm efficacy of catechol, various microscopic techniques viz., light and fluorescence microscopic analyses were carried out ${ }^{28}$. Briefly, the biofilm formation was initiated by growing $C$. albicans on $1 \mathrm{~cm}^{2}$ glass slide in 24-well MTPs containing spider medium with catechol at the concentration of 64,128 and $256 \mu \mathrm{g} / \mathrm{mL}$ for $48 \mathrm{~h}$. After incubation, non-adherent planktonic cells were removed by washing with sterile PBS and then adhered biofilms on the slides were stained accordingly with the type of the microscopic technique undergone. To envisage biofilm under the light microscope, the adhered biofilm cells on the glass slides stained for 5 min with $0.4 \%$ crystal violet. Any excess crystal violet stain was removed by washing with sterile water. After drying, the glass slides were visualized under light microscope at $200 \times$ magnification to examine the differential architecture of formed biofilm (Nikon Eclipse 80i, Japan). To visualize biofilm architecture under fluorescence microscopy, the biofilm formed glass slides were stained by incubation at dark condition for 5 min with $0.1 \%$ acridine orange and excess dye was washed using sterile PBS. Then, the architecture of biofilm was observed under fluorescence microscopy (LSM 710, Carl Zeiss, and Germany).

\subsection{LIVE/DEAD cell viability assay}

To further ascertain the non- fungicidal efficacy of catechol on $C$. albicans sessile cells, LIVE/DEAD analysis was performed using propidium iodide $(\mathrm{PI})$ and acridine orange (AO) staining ${ }^{27}$. After $48 \mathrm{~h}$ treatment of $C$. albicans biofilm with catechol at concentration of 64,128 and $256 \mu \mathrm{g} / \mathrm{mL}, 1 \mathrm{~cm}^{2}$ glass slide were stained using AO/PI stains (Sigma Aldrich) for $15 \mathrm{~min}$ at dark condition. After washing the glass pieces using phosphate buffered saline, the stained cells were visualized under fluorescence microscopy (LSM 710, Carl Zeiss, and Germany) with excitation wavelengths of 525 and $490 \mathrm{~nm}$ for AO and PI, respectively.

\subsection{Yeast-Hyphae $(\mathrm{Y}-\mathrm{H})$ inhibition assay in liquid media}

To assess the influence of catechol on yeast to hyphal transition of $C$. albicans, hyphal growth assay in liquid media was performed as suggested by Bar-Yosef et al., (2017) ${ }^{29}$. In brief, an overnight culture of $C$. albicans was used to inoculate a $1 \mathrm{~mL}$ of spider broth supplemented with different concentrations of catechol (0-1024 $\mu \mathrm{g} / \mathrm{mL}$ ) and incubated for $24 \mathrm{~h}$ at $37^{\circ} \mathrm{C}$. After incubation, the planktonic growth in the spent medium was measured at OD $600 \mathrm{~nm}$. Then, the pellets were resuspended in $10 \mu \mathrm{L}$ of PBS and the ratio of yeast to hyphae cells were further examined via light microscope at $200 \times$ magnifications (Nikon Eclipse 80i, Japan). Phase contrast micrographs of control and treated cells were taken using fluorescence microscopy (LSM 710, Carl Zeiss, and Germany). 


\subsection{Examination of $C$. albicans colony morphology on solid media}

To scrutinize the protrusion of hyphae in the presence and absence of catechol, colony morphology assay was performed on solid spider media ${ }^{30}$. Briefly, $5 \mu$ of $C$. albicans culture was placed on the spider agar medium supplemented with different concentrations $(64,128$ and $256 \mu \mathrm{g} / \mathrm{mL})$ of catechol. Plates were then incubated for 5- 6 days to allow hyphal induction. Then after, the plates were imaged using gel documentation system (GelDoc XR+, Bio-Rad, United States).

\subsection{Lipase assay}

The impact of catechol on the production of lipase was measured by both quantitative and qualitative methods ${ }^{31}$. In qualitative measurement, tributyrin agar medium consists of peptone $0.8 \%$, yeast extract $0.4 \%$, $\mathrm{NaCl} 0.3 \%$ and agar $1.8 \%$ was prepared by autoclaving at $121^{\circ} \mathrm{C}$ for $30 \mathrm{~min}$. After autoclaving, tributyrin $0.2 \%$ and catechol (at 64,128 and $256 \mu \mathrm{g} / \mathrm{mL}$ ) were added to the medium at $43-46^{\circ} \mathrm{C}$. Afterwards, $5 \mu \mathrm{L}$ of overnight C. albicans culture was placed on the center of agar plate and incubated at $37^{\circ} \mathrm{C}$ for $2-3$ days. After incubation, lipase production was examined by measuring zone of clearance around colony using Hiantibiotic zone scale (Himedia, Mumbai). For quantitative assessment, P- Nitrophenyl palmitate (PNP) was used as a substrate. Briefly, the supernatant of $24 \mathrm{~h}$ catechol treated and untreated cells were harvested by centrifugation at 8000 rpm for $10 \mathrm{~min}$. Then, $0.1 \mathrm{~mL}$ of culture supernatant was mixed with $900 \mu \mathrm{L}$ of substrate containing $0.1 \mathrm{~mL}$ of substrate $A(0.3 \% \mathrm{PNP}$ ) and $0.9 \mathrm{~mL}$ of substrate $\mathrm{B}$ (consisting $0.2 \%$ of sodium deoxycholate and $0.1 \%$ of gummi arabicum in $20 \mathrm{mM}$ tris buffer). This reaction mixture was incubated at room temperature for $2 \mathrm{~h}$. After incubation, supernatant was collected and at read at $410 \mathrm{~nm}$.

\subsection{Secreted proteinases assay}

The effect of catechol in the secretion of proteinase was evaluated by both qualitative and qualitative methods demonstrated by Akcaglar et al., (2011), with minor modifications ${ }^{32}$. To qualitatively assess the proteinase secretion, spider medium containing $1 \%$ glucose, $0.05 \% \mathrm{MgSO}_{4}, 2 \%$ agar and $1 \%$ of bovine serum albumin (BSA) in the presence and absence of catechol at 64,128 and $256 \mu \mathrm{g} / \mathrm{mL}$ were prepared. $5 \mu \mathrm{L}$ of $C$. albicans cells $\left(1 \times 10^{6} \mathrm{CFU} / \mathrm{mL}\right)$ was placed on the center of agar plate and incubate at $37^{\circ} \mathrm{C}$ for $2-3$ days. Consecutively, the proteinase production was calculated by measuring the white precipitated zone around colony using Hiantibiotic zone scale (Himedia, Mumbai). In addition, the plates were imaged using gel documentation system (GelDoc XR+, Bio-Rad, United States). To quantify the proteinase production, C. albicans was grown in YEPD medium supplemented with catechol at concentration of 64,128 and $256 \mu \mathrm{g} / \mathrm{mL}$ for $24 \mathrm{~h}$. After incubation, the cell free supernatant was collected through centrifugation at $8000 \mathrm{rpm}$ for $10 \mathrm{~min}$. Then, $0.1 \mathrm{~mL}$ of culture supernatant was mixed with citrate buffer consisting $0.2 \%$ of BSA. After incubation at room temperature for $10 \mathrm{~min}$, the supernatant was read at $280 \mathrm{~nm}$.

\subsection{Cell surface hydrophobicity assay}

Initially, C. albicans was grown for $24 \mathrm{~h}$ with and without catechol at the concentrations of 64, 128 and 256 $\mu \mathrm{g} / \mathrm{mL}^{33}$. Then, the cells were resuspended with YEPD medium to obtain an OD of 1.0. Subsequently, $1 \mathrm{~mL}$ of toluene (SRL, India) was added to each cell suspensions and vortexed vigorously for $1 \mathrm{~min}$. The tubes were kept 
at room temperature for 30 min to allow phase separation. Then, the lower aqueous phase was carefully separated and transferred to fresh polystyrene plate. OD was read at $600 \mathrm{~nm}$. The OD value of strain in the YEPD broth without toluene was used as negative control. The relative hydrophobicity index was expressed as adherence to toluene and was calculated using the following formula: [1- (OD600 nm after vortexing/ OD600 $\mathrm{nm}$ before vortexing) $\times 100$.

\subsection{Preformed biofilm disruption assay}

The preformed biofilm disruption ability of catechol was examined using the crystal violet staining method ${ }^{34}$. For preformed biofilms, the overnight $C$. albicans culture was used to inoculate in 24-well MTPs containing spider medium and incubated at $37^{\circ} \mathrm{C}$ for $48 \mathrm{~h}$. After removal of spent medium, fresh spider broth was added into MTPs along with catechol at varied concentrations $(0-1024 \mu \mathrm{g} / \mathrm{mL})$ and incubated at $37^{\circ} \mathrm{C}$ for $24 \mathrm{~h}$. After incubation, the biofilm formations were spectroscopically quantified at $570 \mathrm{~nm}$. The percentage of biofilm inhibition was calculated using the above mentioned formula.

\subsection{Quantification of C. albicans exopolysaccharide}

To assess the impact, the catechol on $C$. albicans exopolysaccharide production, phenol- sulphuric acid method was performed ${ }^{35}$. Briefly, C. albicans was grown for $24 \mathrm{~h}$ in the presence and absence of catechol at the concentration of 64,128 and $256 \mu \mathrm{g} / \mathrm{mL}$. After incubation, the cell pellet was obtained through centrifugation at $8000 \mathrm{rpm}$ for $10 \mathrm{~min}$. Then, $1 \mathrm{~mL}$ of $0.9 \%$ saline was used to resuspend each cell pellet. An equal volume of $5 \%$ phenol was added to the suspension, followed by 5 volumes of concentrated sulphuric acid. After incubation at room temperature for $1 \mathrm{~h}$ in a dark condition, supernatant was read at $490 \mathrm{~nm}$.

\subsection{Extraction of C. albicans Exopolymeric substances (EPS)}

EPS play an inevitable role in rendering resistance to the sessile cells of $C$. albicans against antimicrobial agents and also act like a protective sheath against host immune response ${ }^{36}$. Hence, the influence of catechol in the production of EPS by sessile cells of $C$. albicans was assessed using the previously stated protocol by Badireddy et al., (2010) ${ }^{37}$. EPS was extracted from both untreated control and catechol treated biofilm cells. In brief, $C$. albicans cells $\left(1 \times 10^{6} \mathrm{CFU} / \mathrm{mL}\right)$ were used to inoculate in YEPD medium supplemented with $10 \%$ of FBS in the presence and absence of catechol (at BIC), and incubated at $37^{\circ} \mathrm{C}$ for $8 \mathrm{~h}$. Subsequent to incubation, the cell-free culture supernatant (CFCS) was separated by centrifugation at $12000 \mathrm{rpm}$ for $10 \mathrm{~min}$. In order to extract cell bound EPS, the pellet so obtained was suspended in $10 \mathrm{~mL}$ of isotonic buffer consisting of $10 \mathrm{mM}$ Tris/ $\mathrm{HCl} \mathrm{pH} \mathrm{8.0,10} \mathrm{mM} \mathrm{EDTA} \mathrm{\&} 2.5 \% \mathrm{NaCl}$, and incubated for overnight at $4^{\circ} \mathrm{C}$. After incubation, the suspension was subjected to centrifugation at $12000 \mathrm{rpm}$ for $10 \mathrm{~min}$. The resulting supernatant (cell-bound EPS) was mixed with already collected CFCS. Now, the pooled EPS (both cell-bound and secreted) was precipitated for overnight at $-20^{\circ} \mathrm{C}$ with double the volume of chilled ethanol. After precipitation, the pelleted form of EPS was obtained by centrifugation for $10 \mathrm{~min}$ at $12000 \mathrm{rpm}$ and stored at $4^{\circ} \mathrm{C}$ until further quantification.

\subsection{Fourier transforms infrared (FTIR) spectroscopic analysis of C. albicans EPS}

The alteration of EPS components upon treatment with catechol were examined using FTIR (Nicolet iS5 FT-IR Spectrometer, Thermo Scientific, USA) ${ }^{31}$. The extracted EPS was mixed with $\mathrm{KBr}$ pellet at the ratio of 1:100 and 
$100 \mathrm{~kg} \mathrm{~cm}^{-2}$ pressure was applied for $5 \mathrm{~min}$ to obtain the pellet from the mixture. Both control and treated cells were scanned in the range of $4000-400 \mathrm{~cm}^{-1}$ with $4 \mathrm{~cm}^{-1}$ resolution. The obtain $\mathrm{KBr}$ pellet spectrum was subtracted from all spectra.

\subsection{Extraction and quantification of $C$. albicans ergosterol}

The total ergosterol content from $C$. albicans was quantified using the standard method suggested by Arthington-Skaggs et al., (1999) with little changes ${ }^{38}$. Briefly, overnight $C$. albicans was used to inoculate $5 \mathrm{~mL}$ of YEPD medium with and without catechol (at BIC) and incubated at $37^{\circ} \mathrm{C}$ for $24 \mathrm{~h}$. Then the culture suspension was centrifuged at 2,700 rpm for $5 \mathrm{~min}$ to harvest the stationary-phase cells. Then, the cell pellet was dissolved with $300 \mu \mathrm{L}$ of $25 \%$ alcoholic potassium hydroxide solution and vortexed vigorously for $1 \mathrm{~min}$. Next, the tubes were incubated in water bath at $85^{\circ} \mathrm{C}$ for $1 \mathrm{~h}$. After allowing the tubes to cool at room temperature, $400 \mu \mathrm{L}$ of mixture containing sterile distilled water and $n$-heptane (SRL, India) in the ratio of 1: 3 was added to each tube, and subjected to vigorous vortexing for $3 \mathrm{~min}$. Now, the top $\mathrm{n}$-heptane layer entrapping ergosterol was transferred to fresh microfuge tubes and stored at $-20^{\circ} \mathrm{C}$ for $24 \mathrm{~h}$. Before the spectral analysis, a $100 \mu \mathrm{L}$ extracted sterol was diluted with five-fold $100 \%$ ethanol and then scanned spectrophotometrically between 240 and $300 \mathrm{~nm}$.

\subsection{Extraction and FTIR analysis of farnesol}

The farnesol was extracted from C. albicans using a method demonstrated by Hornby et al., (2004) with necessary modifications ${ }^{39}$. In brief, $1 \times 10^{6}$ of $C$. albicans cells was used to inoculate $40 \mathrm{~mL}$ of GPP medium (20 g glucose, $6 \mathrm{~g} \mathrm{Na}_{2} \mathrm{HPO}_{4}$. $7 \mathrm{H}_{2} \mathrm{O}, 4 \mathrm{~g} \mathrm{KH}_{2} \mathrm{PO}_{4}, 0.5 \mathrm{~g} \mathrm{MgSO}_{4} .7 \mathrm{H}_{2} \mathrm{O}, 1 \mathrm{mg} \mathrm{CuSO}$. $5 \mathrm{H}_{2} \mathrm{O}, 1 \mathrm{mg} \mathrm{ZnSO}$. $7 \mathrm{H}_{2} \mathrm{O}, 1 \mathrm{mg}$ $\mathrm{MnCl}_{2}, 1 \mathrm{mg} \mathrm{FeSO}_{4}, 20 \mu \mathrm{g}$ biotin, $200 \mu \mathrm{g}$ pyridoxine $\mathrm{HCl}, 200 \mu \mathrm{g}$ thiamine $\mathrm{HCl}$ and $10 \mathrm{mM}$-proline) in the presence and absence of catechol (at BIC). Tubes were incubated at $37^{\circ} \mathrm{C}$ for $24 \mathrm{~h}$ under shaking condition. After incubation, the cell suspension was centrifuged at $12000 \mathrm{rpm}$ for $20 \mathrm{~min}$. Then, the culture supernatant was filter sterilized using $0.2 \mu \mathrm{m}$ Whatman cellulose nitrate filters affixed in vacuum filtration apparatus. The resulting CFCS was then extracted with $10 \mathrm{~mL}$ of ethyl acetate. Dried residues were suspended with $1 \mathrm{~mL}$ of 20

$\%$ ethyl acetate- hexane mixture and transferred to fresh microfuge tubes. Next, the residual solvents were removed under vacuum evaporator. Then, the changes in farnesol production upon treatment with catechol were investigated using FTIR (Nicolet iS5 FT-IR Spectrometer, Thermo Scientific, USA). The extracted farnesol from both catechol treated and untreated cells suspension were mixed with KBr pellet at the ratio of 1:100 and pelleted form of mixture was obtained by applying $100 \mathrm{~kg} \mathrm{~cm}^{-2}$ pressure for $5 \mathrm{~min}$. The pellets were scanned in the range of $4000-400 \mathrm{~cm}^{-1}$ with $4 \mathrm{~cm}^{-1}$ resolution. The obtain $\mathrm{KBr}$ pellet spectrum was subtracted from all spectra.

\subsection{Serial passage experiment}

In order to investigate whether repeated exposure of catechol leads to resistance development in C. albicans, serial passage experiment was carried out as previously demonstrated by Pierce et al., $(2015)^{40}$. Initially, $1 \times 10^{6}$ $\mathrm{CFU} / \mathrm{mL}$ C. albicans cells were used to inoculate $2 \mathrm{~mL}$ of YEPD and spider medium with and without catechol at the hyphal inhibitory concentration of $128 \mu \mathrm{g} / \mathrm{mL}$. Cultures were incubated at $37^{\circ} \mathrm{C}$ for $24 \mathrm{~h}$ with agitation to allow hyphal induction. Every day from then, $20 \mu \mathrm{L}$ from each culture (both control and catechol treated) were serially transferred into $2 \mathrm{~mL}$ of fresh medium (YEPD or spider) supplemented with catechol (at $128 \mu \mathrm{g} / \mathrm{mL}$ ). 
This daily transfer was carried out for 8 days without any interruption and further this was continued for additional 7 days by doubling the concentration of catechol at $256 \mu \mathrm{g} / \mathrm{mL}$. Before daily transfer, cells were visualized under microscope to examine the hyphal inhibition and also $5 \mu \mathrm{L}$ of cultures from each YEPD medium (control and treated) were spotted on the YEPD agar plate to investigate the impact of catechol on planktonic growth of $C$. albicans.

\subsection{Impact of catechol on antifungal efficacy of azole and polyene antifungals}

Before assessing the potency of catechol in increasing the susceptibility of $C$. albicans towards conventional antifungal drugs, the susceptibility of $C$. albicans against flucanozole, ketoconazole, micanazole, amphotericin$B$ and nystain were first determined through microbroth dilution assay as explained earlier ${ }^{41}$. The MIC of each antifungal drug against $C$. albicans was determined spectroscopically at OD $600 \mathrm{~nm}$. Next, disc diffusion and micro- broth dilution assays were performed to investigate the efficacy of catechol (at BIC) to enhance the susceptibility of traditional antifungals against $C$. albicans. Briefly, $1 \times 10^{6} \mathrm{CFU} / \mathrm{mL}$ of $C$. albicans was used to inoculate in YEPD medium with and without catechol and incubated at $37^{\circ} \mathrm{C}$ for $3 \mathrm{~h}$. In microbroth dilution assay, $1 \%$ of untreated control and catechol treated $C$. albicans cells added to the YEPD medium supplemented with appropriated MIC of each anti-fungal drugs. After incubation at $37^{\circ} \mathrm{C}$ for $24 \mathrm{~h}$, the growth OD was measured at $600 \mathrm{~nm}$ using spectrophotometer (Spectra Max 3, Molecular Devices, United States). In disc diffusion assay, both the untreated control and catechol treated cultures were swabbed on separate YEPD agar plates, and antifungal loaded discs were placed on the center of the swabbed agar plates. After incubation at $37^{\circ} \mathrm{C}$ for $24 \mathrm{~h}$, difference in zone of clearance around the colonies were measured and documented using high resolution CCD camera (GelDoc XR+, Bio-Rad).

\subsection{RNA preparation, CDNA synthesis and Real Time PCR analysis (at $C$. albicans)}

The differential expression of $C$. albicans'virulence genes upon catechol treatment was evaluated using Real Time PCR (qPCR) analysis ${ }^{34}$. The total RNA from the control and catechol treated (at $256 \mu \mathrm{g} / \mathrm{mL}$ ) C. albicans cells were extracted using Trizol method and quantified by the nano spectrophotometer (Shimadzu, Japan). Subsequently, cDNA were constructed from the isolated RNA $(1 \mathrm{mg} / \mathrm{mL})$ using High capacity cDNA Reverse Transcription kit (Applied Biosystems, USA). qPCR was performed for the three positive (RAS1, HWP1, ALS3) and two negative regulator genes (NRG1, TUP1) involved in hyphae and biofilm formation. The primers were included individually along with cDNA and SYBR Green kit (Applied Biosystems, USA) at the final reaction volume of $10 \mu \mathrm{L}$. The qPCR analysis of selected genes was carried using the thermal cycler (7500 Sequence Detection System). The sequence and functions of the used genes are tabulated in Table. 1. The expression profile of selected genes was normalized using $C$. albicans'ITS region. The fold change in gene expressions was quantified by $2^{-\triangle \Delta C T}$ method.

\subsection{Statistical Analysis}

All the experiments were carried out in biological triplicates with at least two experimental replicates and the data were presented as mean \pm standard deviation. To evaluate statistical differences between control and treated samples one-way analysis of variance (ANOVA) and Dunnett's post hoc test was performed using SPSS statistical software 17.0. The significance was represented as $p \leq 0.05$ and $<0.01$, respectively. 


\section{Results \& Discussion}

Over a long period of time, the term drug resistance has been travelling with us owing to the continuous exposure of antimicrobial agents in the fight against infectious diseases. Mechanistically, most of the antifungal drugs administered till date in clinical settings have been tend to render more selective pressure which perpetuates drug resistance. This would lead us to a 'no drug of last resort' situation, before which, a promising alternative way to combat drug- resistant pathogens should be evolved. Antivirulence being the best among few alternative therapies, in the present study attempts was made to explore an antivirulence compound from plant resources against drug-resistance $C$. albicans, which cause deadly diseases in immunocompromised patients. As traced to antiquity, plants and plant-derived natural compounds have been playing a pivotal role in medical treatments. In recent days, phytochemicals with their pharmaceutical ability to selectively target the virulence attributes at modest consequences have utterly grabbed the researchers' attention as an antivirulent agent ${ }^{42}$. Among these phytochemicals, we found catechol, one of the simplest allelochemicals rich in onions, maple and oak, as the promising antivirulence agents targeting biofilm and hyphal formation in C. albicans. Despite potent broad-spectrum antimicrobial efficacy against various pathogen $\mathrm{s}^{19}$, the present study is the first of its kind to layout the inhibitory propensity of catechol at sub-lethal concentrations against different virulence attributes of $C$. albicans. Primarily, a wide range of catechol concentrations were deployed to identify its MIC against $C$. albicans ATCC 10231, and it was determined to be $1024 \mu \mathrm{g} / \mathrm{mL}$. An earlier report by Kocaçalışkan et al., (2006) demonstrated the antimycotic effect of catechol against Fusarium oxysporum and Penicillium italicum; nevertheless, the data of the present study for the first time unveiled its anticandidal efficacy ${ }^{18}$.

\subsection{Catechol at sub-MICs does not affect the basic metabolic function of $C$. albicans}

Since, the fundamental phenomenon of antivirulent is that it should not have any influence on basic metabolism of pathogen, a great deal of attention has been given to evaluate the non-fungicidal effect of catechol at used concentrations against $C$. albicans viability. The obtained result of microbroth dilution assay showed that the sub-MICs of catechol $(0-256 \mu \mathrm{g} / \mathrm{mL})$ did not show any significant impact on the growth of planktonic cells (Fig. 1A). However, the higher concentration of catechol i.e. $1024 \mu \mathrm{g} / \mathrm{mL}$ reduced the $C$. albicans growth to a significant level $(P<0.01)$. Further, to substantiate the non-fungicidal effect of catechol at sub-MICs, alamar blue assay was performed. Here, an oxidation-reduction (REDOX) indicator resazurin was used to indicate the metabolically active cells. A dose-dependent decrease in the intensity of the pink color or reduced resorufin during catechol treatment was observed, which is depicted in the Fig. 1B. The intensity of pink color produced by the cells exposed with catechol at concentrations up to $256 \mu \mathrm{g} / \mathrm{mL}$ was very close to the intensity of pink color produced by the control cells. However, the retained blue color by the cells exposed with high concentrations (512 and $1024 \mu \mathrm{g} / \mathrm{mL}$ ) of catechol showed the un-metabolized state of resazurin, which in turn ascertained high concentration -mediated anticandidal effect of catechol. Taken together, both the growth OD assay and the alamar blue assay confirmed that the catechol at sub-MICs does not exert any negative impact on the growth and viability of $C$. albicans even after $24 \mathrm{~h}$ incubation. Hence, below sub-MICs of catechol (0-256 $\mu \mathrm{g} / \mathrm{mL}$ ) were considered for further analysis to evaluate the antivirulence potential against $C$. albicans. 


\subsection{Catechol at sub-MIC deteriorates yeast-hyphal transition of C. albicans}

Pathogenesis of $C$. albicans is attributed to diversified virulence secretions which include, environmental adaptation factors, adhesins, yeast to hyphal transition, secreted enzymes, phenotype switching and biofilm formation ${ }^{43}$. Amongst, yeast to hyphal transition is considered as one of the putative virulence traits, as it coordinately regulates the other virulence traits for host cellular invasion ${ }^{29}$. By placing the NRG1 gene (a negative regulator of yeast to hyphae transition) into tetracycline promoter for differential regulation of hyphal formation (switch on and off), a research group have testified and ascertained that hyphal form of $C$. albicans is solely responsible to attribute for the mortality during disseminated candidal infections ${ }^{44}$. More other investigators from worldwide have suggested that the molecules with profound efficacy to inhibit yeast to hyphae transition represent an attractive candidate for antivirulence agents ${ }^{29,34}$. Hence, to examine the antihyphal efficacy of catechol at varied concentrations $(0-1024 \mu \mathrm{g} / \mathrm{mL})$, we performed hyphal inhibitory assay in liquid spider medium. When cultured in liquid spider medium, the light micrographs of untreated control groups exposed a dense network of twig-like hyphal formation (Fig. 2). On the other hand, the micrographs of catechol treated groups displayed evenly scattered yeast cells (Fig. 2). Furthermore, the spectroscopic reading of spent spider media also revealed that the catechol does not have any impact on $C$. albicans planktonic growth. This clearly signified the antihyphal efficacy of catechol without posing any negative impact on the metabolic function of both yeast and hyphal cells (Fig. 2). To further analysis the influence of catechol on other virulence traits of $C$. albicans, narrow range of catechol concentration $(64,128$, and $256 \mu \mathrm{g} / \mathrm{mL})$ were used.

\subsection{Catechol at sub- MIC inhibits the $C$. albicans hyphal morphology in solid media}

Besides, to know the effect of catechol on $C$. albicans hyphal morphology, spot assay was employed. The overnight culture of $C$. albicans spotted on solid spider medium treated with catechol depicted a smooth colony with profoundly reduced hyphal protrusions at the periphery. On the other hand, the untreated control plates showed the colony characteristics of a typical virulent $C$. albicans i.e. rough colony with dense radius of lengthy hyphal projection (Fig. 3). A gradual reduction in the hyphal protrusion was witnessed with the increasing concentrations of catechol $(64,128$ and $256 \mu \mathrm{g} / \mathrm{mL})$. This is in line with the results of earlier report by Pierce et al., (2015) wherein, structural analogs of diazaspiro-decane exhibited a concentration dependent hyphal inhibitiory efficacy against $C$. albicans hyphal growth. Both the solid and liquid assays data distinctly unveiled the excellent hyphal inhibitory efficacy of catechol ${ }^{40}$.

\subsection{Catechol at sub-MIC deconstructs the architecture of $C$. albicans biofilms}

In general, it is anticipated that the molecules which modulate $C$. albicans yeast to hyphae transition could potentially interrupt the biofilm assemblage owing to the close association between hyphae and biofilms. Further, understanding the clinical significance of biofilm in the emergence of drug resistance, as it renders a safe environment to the residing planktonic cells from the devastating action of antimicrobial agents, the in vitro antibiofilm efficacy of catechol at varied concentrations $(0-1024 \mu \mathrm{g} / \mathrm{mL})$ was assessed by employing crystal 
violet quantification method ${ }^{31}$. The spectroscopic quantification (at OD $570 \mathrm{~nm}$ ) of C. albicans biofilm biomass grown under a varied range of catechol concentrations $(32,64,128$ and $256 \mu \mathrm{g} / \mathrm{mL})$ revealed its dosedependent antibiofilm activity (Fig. 4A). As $256 \mu \mathrm{g} / \mathrm{mL}$ of catechol exhibited a maximum of $93 \%$ reduction in $C$. albicans biofilm biomass, this concentration was fixed as BIC. It is also notable that the very low concentration of catechol $(32 \mu \mathrm{g} / \mathrm{mL})$ was effectively control $30 \%$ of $C$. albicans biofilms. The representative plate image of biofilm inhibition by catechol was shown in Fig. 4B.

\subsection{In situ microscopic examinations authenticate the biofilm obliteration upon catechol treatment}

To comprehensively inspect the catechol mediated biofilm destruction on glass surfaces, light and fluorescence microscopic analyses were performed (Fig. 5). Both the light and fluorescence micrographs of untreated control unveiled a robust crisscross architecture of biofilm encompassing both yeast and long filamentous hyphal cells. In contrast, this characteristic of biofilm architecture was immensely collapsed in a concentration-dependent manner upon $24 \mathrm{~h}$ of treatment with catechol. Significant inhibition of micro-colony and hyphal formation was found in C. albicans treated with catechol at $256 \mu \mathrm{g} / \mathrm{mL}$. Likewise, a notable biofilm inhibition was observed at the concentrations of 64 and $128 \mu \mathrm{g} / \mathrm{mL}$. Microscopic examinations on a whole depicted that catechol attenuates planktonic cells towards forming biofilm architecture in a dose-dependent fashion.

\section{6. Catechol at sub-MIC does not kill C. albicans sessile cells}

To investigate true viability of $C$. albicans biofilm cells under treatment with catechol, LIVE/ DEAD analysis was performed. AO is a cell permeable dye; hence it can stain the live cells. However, PI can stain the dead cells. As depicted in Fig. 6, there is no difference was observed in catechol treated and untreated control samples. The merged microscopic image of control sample revealed the presence of live cells more than the dead cells.

Likewise, the cells treated with catechol at the concentration of $64,128,256 \mu \mathrm{g} / \mathrm{mL}$ exposed the more number of live cells than dead cells. This assay further affirmed that catechol reduced the $C$. albicans biofilm formation without hampering its growth.

\subsection{Catechol at sub-MIC effectively hampers $C$. albicans virulence secretions}

To gain more insight into the antibiofilm and antihyphal action of catechol, differential expression of various virulence traits upon supplementation with catechol was investigated. As depicted earlier, C. albicans secretes different extracellular hydrolytic enzymes, which facilitate cellular invasion by proficiently degrading the host's proteins and cell membrane ${ }^{45}$. Since, secreted aspartyl proteinases (Sap) and lipase of C. albicans are being the most discussed extracellular hydrolytic enzymes, the in vitro inhibitory potency of catechol at BIC was assessed against these two virulence enzyme production. Inhibition of $C$. albicans aspartyl proteinase was found to be an attractive strategy to modulate its pathogenesis. For instance, Hoegl et al., (1998) reported that HIV patients who have undergone treatment with HIV protease inhibitors showed resistance to mucosal candidiasis, as the HIV protease inhibitors significantly hampered the production of secretary aspartic proteases from $C$. albicans upon infection $^{46}$. Similarly, it has been reported that compounds that possess the anti-lipase efficacy showed a broad spectrum of antifungal activity against different kinds of pathogenic fungi especially C. albicans, Cryptococcus 
neoformans and Aspergillus flavus ${ }^{47}$. Thus, the catechol was taken forward to explore its impact on $C$. albicans virulence scenery such as lipase and protease productions. When $C$. albicans cells were treated with BIC of catechol, both proteolytic and lipolytic zone around the fungal colony were found to be significantly abridged when compared to their respective untreated controls. In protease production assay, white precipitated zone of untreated control plate was found to be $10 \mathrm{~mm}$, whereas, the plates incorporated with varied catechol concentrations 64,128 and $256 \mu \mathrm{g} / \mathrm{mL}$ displayed the zones of reduced diameter of $5.5,5$ and $3.5 \mathrm{~mm}$, respectively (Fig. 7B). Similarly, the lipolytic transparent zone in lipase assay was found to be $3.5 \mathrm{~mm}$ in the control plate. On the other hand, the catechol treated plates at concentrations 64,128 and $256 \mu \mathrm{g} / \mathrm{mL}$ unveiled a dose-dependent reduction in zone formation i.e. 3.5, 3 and $2 \mathrm{~mm}$, respectively (Fig. 7D). The lipase and protease productions were also quantified using PNP and BSA as substrate, respectively. The data obtained from spectroscopic reading further confirmed that $C$. albicans cells with catechol at the concentrations of 64,128 and $256 \mu \mathrm{g} / \mathrm{mL}$ showed reduced lipase and protease productions compared to untreated control cells (Fig. 7A\&C).

\subsection{Catechol treatment potentially diminishes $C$. albicans' surface hydrophobicity}

Cellular hydrophobicity is an important phenomenon as it positively correlates adhesion and biofilm formation of microbial cells and attachment surface (biotic or abiotic), which in sequence increases the virulence of the pathogen ${ }^{48}$. In this current study, the classic MATH assay was performed to evaluate the potency of catechol on the hydrophobic interaction of $C$. albicans with toluene (non-polar solvent) (Fig. 8). After incubation, the hydrophobic index of catechol treated and untreated control cells were evaluated through vortexing with toluene. Phenomenally, catechol has a potent ability to lessen $C$. albicans hydrophobicity in a dose-dependent fashion compared to that of untreated control, which showcases the hydrophobicity index of $63 \%$. Upon treatment with BIC of catechol $(256 \mu \mathrm{g} / \mathrm{ml})$, the hydrophobicity index of C. albicans was considerably abridged to about $42 \%$.

\subsection{MIC of Catechol disintegrates preformed biofilms of $C$. albicans}

Once the biofilms were established (so called mature biofilm), the embedded sessile cells become tremendously vigorous that makes all kinds of antimicrobial agents to be ineffective. Especially, mature biofilms formed by $C$. albicans are highly heterogeneous in nature in terms of their cellular distribution and extracellular material. As mature biofilms are extremely hard to eradicate with high doses of antimicrobials, it also symbolizes to be a source of recurrent infection ${ }^{49}$. Therefore, the impact of catechol on C. albicans'mature biofilm (48 h) was evaluated using crystal violet staining method (Fig. 9). To begin with, the mature biofilm disrupting efficacy of catechol at BIC $(256 \mu \mathrm{g} / \mathrm{ml})$ was evaluated using MTP biofilm biomass assay. Data revealed that BIC of catechol was not sufficient enough to pose any impairment on the preformed biofilms of $C$. albicans. Hence, out of curiosity, we deployed MIC of catechol to treat against $48 \mathrm{~h}$ preformed biofilms. As anticipated, the MIC of catechol proficiently disintegrated the preformed biofilm up to about $68 \%$. Hence, it is speculated that the antibiofilm mechanism of catechol (at BIC) could plausibly be due to the hindrance of principal regulators that needed for the initial biofilm assemblage i.e. yeast to hyphal transition and cellular hydrophobicity. Consequently, manifestation with catechol at BIC was not adequate to disrupt and disintegrate the preformed biofilms, as they are highly adhered and enriched with dense network of existing filamentous cells. This result 
correlates well with the observation by sivaranjani et al., (2016), wherein BIC of morin was not sufficient to disrupt the preformed biofilms of Listeria monocytogenes, however, it exhibited a antibiofilm efficacy through blocking initial attachment ${ }^{50}$.

\section{10. Catechol at sub-MIC reduces the $C$. albicans exopolysaccharide production}

The impact of catechol on the production of exopolysaccharide was quantified by measuring total carbohydrate content by phenol sulfuric acid method. The spectroscopic data revealed that catechol reduced the total carbohydrate content in concentration depended manner (Fig. 10). A significant reduction of carbohydrate content was observed at the catechol concentration of $256 \mu \mathrm{g} / \mathrm{mL}(P<0.01)$.

\subsection{Catechol at BIC mediates alteration in EPS components of C. albicans' biofilms}

The sessile cells are enmeshed in a complex network of hydrated matrix known as EPS, which creates a microenvironment that hinders the penetration of antifungal drugs either through sequestering it or by modulating the degrading enzymes. It is also noted that a maximum of $90 \%$ biofilm dry weight accounted for the hydrated EPS matrix, and the remaining portions are occupied by planktonic cells ${ }^{51}$. Therefore, FTIR analysis in the range of $4000 \mathrm{~cm}^{-1}-400 \mathrm{~cm}^{-1}$ was performed to understand the catechol-mediated EPS modifications at the biomolecular level, if any. FTIR spectral analysis is a well-known technique commonly used to identify the modifications in EPS components, especially carbohydrates, proteins and lipids. Through the FTIR spectra, various parameters were taken into account to distinguish the cellular compositions of biological samples such as the difference in peak position, bandwidth and band intensity. In the IR spectrum of catechol treated (at 256 $\mu \mathrm{g} / \mathrm{mL}$ ) sample, the shape and intensity of the absorbance peak were considerably reduced contrast to the spectrum of untreated control. Substantial difference in EPS components under catechol treatment was quite obvious at three spectral regions including (i) $3600-3000 \mathrm{~cm}^{-1}$, (iii) $1800-1500 \mathrm{~cm}^{-1}$ and (iv) $800-400 \mathrm{~cm}^{-1}$ (Fig. 11) corresponding to the absorptions of lipids, amide bonds of proteins \& peptides and polysaccharides, respectively. The catechol mediated decrease in the peak corresponding protein could be attributed to the phenomenal suppression of virulence protein secretion under the presence of catechol. Thus, it is envisaged that catechol could potentially reduce the biofilm assemblage of $C$. albicans by plausibly targeting the pathways driving the synthesis of extracellular proteins and polysaccharides. Further, catechol with its EPS modulating efficacy, it is also anticipated to effectively increase the vulnerability of $C$. albicans towards the active penetration and action of conventional antifungals.

\subsection{Manifestation of catechol does not dwindles the ergosterol production in C. albicans}

Ergosterol is the basal component in the fungal cell membrane and plays a crucial role in their permeability and fluidity. Due to its unique function, ergosterol served as a target for most of the conventional antifungal drugs, for instance, polyenes and azoles. The drug resistance to these antifungals has also been reported as they provoked selection pressure by targeting ergosterol ${ }^{52}$. This circumstance demands us to examine the impact of catechol on ergosterol production using UV spectrophotometer. The occurrence of four representative peaks between 240 and $260 \mathrm{~nm}$ in UV spectra affirmed the presence of ergosterol and other sterol intermediates in the extracted samples (Fig. 12). The UV spectral profile of control and treated samples displayed peaks of very similar intensity, which proves that the catechol treatment does not pose any impact on the production of ergosterol. On a contrary to our finding, the treatment with antivirulence drug combinations (quinic acid and 
undecanoic acid) reduced the production of ergosterol as demonstrated in the report by muthamil et al., $(2018)^{31}$. Since, the ergosterol biosynthetic pathway is not being targeted by catechol, it is appropriate to state that catechol would not mediate resistance development.

\subsection{Catechol triggers the production of farnesol -the quorum sensing molecule of $\mathrm{C}$. albicans}

The hyphal formation in $C$. albicans necessarily involves two important pathways namely, Ras-dependent (RascAMP-PKA) and Ras-independent (Ubr1-Cup9).As far as the pathogenesis and infection by C. albicans are

concerned, yeast to hyphal transition has been mediated through the Ras-cAMP-PKA pathway ${ }^{53}$. In $C$. albicans, farnesol is the quorum sensing molecule, which naturally blocks the yeast to hyphae transition at relatively higher concentrations. At high cell density, the increased farnesol production negatively regulates Ras-cAMPPKA signaling pathway by attenuating the Ras1 binding towards Ras associated domain of adenylate cyclase (cyr1) [positive regulator of yeast to hyphal switching] ${ }^{54}$. In order to affirm that the observed antihyphal activity might be due to increased farnesol production, FTIR analysis was performed (Fig. 13). The detected peaks were compared with the FTIR library search to confirm the presence of farnesol. The obtained FTIR spectral profile certainly showcases that the absorbance peak of treated was two-fold higher than the absorbance peak of control. Thus, it is envisaged that the mechanism of antihyphal action of catechol could be the activation of quorum sensing molecule in C. albicans i.e. farnesol. In par with this, a study by Hornby \& Nickerson (2004) demonstrated a farnesol-induced antifungal efficacy of azole drugs against $C$. albicans, however, the current study claims for farnesol-induced antihyphal efficacy of a phytochemical ${ }^{39}$.

\subsection{C. albicans does not exerts resistant menace against catechol's repeated exposure}

As described earlier, drug-resistant strains have been constantly increasing owing to the continuous exposure of antimicrobials, which lead to cause conflicting results compared with susceptible strains ${ }^{15}$. This issue guides us to investigate whether continuous exposure with catechol would induce the development of resistance in $C$. albicans. Hence, the serial passage experiment was performed to evaluate the possibility of spontaneous resistance development upon continuous exposure with catechol as previously demonstrated by Pierce et al., $(2015)^{40}$. Initially, C. albicans populations were exposed to catechol at its hyphal inhibitory concentration (128 $\mu \mathrm{g} / \mathrm{mL}$ ) for 8 days in both YEPD and spider medium. Then, the concentration of catechol was increased up to twofold $(256 \mu \mathrm{g} / \mathrm{mL})$ for further 7 days. The populations in the spider medium were continuously monitored for hyphal inhibition under the light microscope every day (Fig. 14A). The micrographs of untreated control cells bare the deep and elongated hyphal formation. As expected, the catechol-treated $C$. albicans cells displayed evenly distributed yeast cells even after 15 days of serial passage. These results evidently demonstrated the long-lasting anti-hyphal efficacy of catechol under subsequent treatment for 15 days. Besides, the cells treated with catechol in the YEPD medium were spotted on the YEPD agar plates to investigate the impact of catechol on the metabolic viability of $C$. albicans. The obtained results revealed that the prolonged catechol manifestation did not display any marked effect on the growth of C. albicans (Fig. 14B). Thus, the serial passage data reinforces that catechol-mediated antivirulence therapy does not induce the spontaneous emergence of resistance.

\subsection{Pretreatment with catechol potentiates the antifungal efficacy of azoles and polyenes}


Combination therapy is one of the effective approaches for the reconsideration of old drugs that are unavailable in medical settings today. Most of the synergistic studies have deliberated that the utilization of antibiofilm agents in combination with antifungal agents would potentially sensitizes the pathogen to antifungal agents ${ }^{55}$. Moreover, recent reports have also illustrated that a combination of antifungal agents with newly identified phytochemicals exhibited more efficacy with low toxicity and a broader spectrum of action ${ }^{2}$. For example, a research group identified that the combined action of eugenol significantly reduced the SMIC (sessile MICs) of fluconazole to 32 -fold ${ }^{56}$. Similarly, quite a few investigators observed that the synergistic therapy by the antibiofilm agents such as chloroquine and cyclosporine also potentiate the susceptibility rate of $C$. albicans to conventional antifungals ${ }^{57}$. In this milieu, we further examined the efficacy of catechol in potentiating the anticandidal activity of known conventional antifungal drugs using microbroth dilution and disc diffusion assay. Primarily, the MICs of used antifungals viz., fluconazole, amphotericin-B, ketoconazole, miconazole and nystatin were identified to be $256,3,16,16$ and $4 \mu \mathrm{g} / \mathrm{mL}$, respectively. As anticipated, the data of microbroth dilution also exposed that pretreatment with catechol has reduced the growth OD compared to untreated control $C$. albicans cells (Fig. 15A). Furthermore, the results of the Kirby-Bauer disc diffusion susceptibility test revealed that $C$. albicans pretreatment with catechol $(128 \mu \mathrm{g} / \mathrm{mL})$ for $12 \mathrm{~h}$ displayed an increased zone of clearance around the discs (loaded with amphotericin-B, ketoconazole, miconazole and nystatin) compared to that of the colony formed by untreated C. albicans (Fig. 15B). However, catechol did not exhibit even a marginal effect over the action of fluconazole. These data signify that the catechol beyond mitigating the hyphal and biofilm formation, it also potentiates the susceptibility of $C$. albicans towards antifungals to a greater extent, which is an excellent pharmaceutical characteristic feature exhibited by a molecule to be an effective alternative to antibiotics.

\subsubsection{Catechol treatment down-regulates the candidate genes of Ras-cAMP-PKA pathway}

To further evaluate the antihyphal and antibiofilm effect of catechol at transcriptomic level, qPCR analysis was performed. As previously discussed, Ras-cAMP- PKA pathway was found to play a vital role than the all other signal transduction pathways in $C$. albicans pathogenesis ${ }^{53}$. In this pathway, RAS1 act as positive regulator binds with CYR1 (adenylate cyclase) and thereby regulates the expression of several virulence genes responsible for filamentation and host cell invasion. On the other hand, NRG1 negatively regulates the hyphal initiation along with the co-repressor $T U P 7^{54}$. With this background, we have selected five candidate genes such as NRG1, TUP1, HWP1, ALS3 and RAS1 involved in Ras-CAMP- PKA pathway. As anticipated, the qPCR data unveiled the down-regulation of hyphae specific genes in the presence of catechol (at BIC). RAS1 is an upstream regulator gene of Ras-CAMP- PKA pathway found to be downregulated upto - 6.3 fold during catechol treatment (Fig. 16). On account of this, the other two downstream activating genes such as HWP1 and ALS3 were also downregulated to -3.6 and -3.4 fold, respectively.

\subsubsection{Catechol triggered farnesol-mediated hyphal inhibition through up-regulation of TUP1}

In addition, one of the negative regulator genes TUP1 was found to be significantly upregulated upto 2.5 fold ( $P$ $<0.05)$. TUP1 was reported to negatively regulate the genes accountable for initiating filamentous growth by interacting with co-repressor NRG1. A study by Kebaara et al., (2008) demonstrated the correlation between the farnesol mediated hyphal inhibition and increased expression of TUP1 mRNA in C. albicans ${ }^{58}$. They have proved that the addition of farnesol leads to increase in TUP1 expression at both transcriptional and translational level, and also found that farnesol was unable to inhibit filamentation in TUP1 mutant stains. In 
comparison with the previous finding, we have suggested that the mode of action of catechol could be plausibly by triggering the quorum sensing molecule - farnesol, which subsequently inhibited the hyphal and biofilm formation in C. albicans.

However, unlike TUP1, the expression profile of another negative regulator NRG1 was downregulated, but not at the significant level $(-0.87$ fold). Studies on molecular pathogenesis of $C$. albicans have demonstrated the disappearance of $N R G 1$ from hyphae specific promoters during the initial $30 \mathrm{~min}$ of hyphal induction ${ }^{54}$. Within 60 min of time the level of NRG1 becomes normal. This could be attributed to the weak fold change in NRG1 expression between catechol treated and untreated control cells. The overall gene expression profile of $\mathrm{C}$. albicans genes under catechol treatment was illustrated in Fig. 17.

\section{Conclusions}

The use of an antivirulent agent, especially plant-derived phytochemicals as alternative to antimicrobial chemotherapy would be an effective measure to control clinically important fungal pathogens. The current research work illustrates the antibiofilm and antihyphal efficiencies of catechol against a predominant fungal pathogen $C$. albicans. Moreover, inferred data divulge that catechol is successful in substantially reducing the secretary virulence enzymes viz., lipase and protease. Besides, the study also confirms that treatment with catechol does not compromise any fundamental biosynthetic components of $C$. albicans specifically ergosterol. Further, it is envisaged that the antivirulence mode of action of catechol against $C$. albicans could be the triggering of quorum sensing molecule -farnesol, whose accumulation naturally hampers hyphal elongation and its prerequisite -biofilm formation through increasing the expression of TUP1 (hyphal negative regulator). The serial passage experiment further reinforced the sustained non-fungicidal -antihyphal efficacy of catechol for a long period of time. Thus, the present study provides new insights for the use of a natural compound -catechol as an alternative remedy to treat drug-resistant $C$. albicans infections.

\section{Declarations}

\section{Author Contributions:}

"Conceptualization, project supervision, S.G.; methodology, validation, formal analysis, writing-original draft preparation, R.J.; writing-review and editing, R.S., P.K., S.K.P., S.G.; Critically revised the original draft, S.K.P.; project administration, funding acquisition, S.G.; All authors have read and agreed to the published version of the manuscript."

\section{Funding:}

SG gratefully acknowledges UGC for Start-Up Grant (Grant No. F.30-381/2017(BSR)/F.D Diary No. 2892) and DST-SERB-EEQ Project Grant (File No.:EEQ/2020/000288). The authors thankfully acknowledge the financial support rendered by RUSA 2.0 [F.24-51/2014-U, Policy (TN Multi-Gen), Department of Education, Government of India].

\section{Acknowledgments:}


The authors thankfully acknowledge DST-FIST [Grant no. SR/FST/LSI-639/2015(C)], UGC-SAP [Grant no. F.5-1/ 2018/DRS-II (SAP-II)], and DST-PURSE [Grant no. SR/ PURSE Phase 2/38 (G)] for rendering instrumentation \& infrastructure facilities. SG gratefully acknowledges UGC for Start-Up Grant (Grant No. F.30-381/2017(BSR)/F.D Diary No. 2892) and DST-SERB-EEQ Project Grant (File No.:EEQ/2020/000288). The authors thankfully acknowledge the financial support rendered by RUSA 2.0 [F.24-51/2014-U, Policy (TN Multi-Gen), Department of Education, Government of India]. RJ thanks to R. Alexpandi, Research Scholar, Department of Biotechnology, Alagappa University, for the support extended during FTIR analysis. RJ sincerely acknowledge Rashtriya Uchchatar Shiksha Abhiyan (RUSA), MHRD, Government of India [F. 24-51/2014-U, Policy (TN Multi-Gen), Dept. of Edn, GOl] for providing RUSA-2.0 Ph.D. Fellowship.

Conflicts of Interest: The authors declare no conflict of interes

\section{References}

1. d'Enfert, C. Hidden killers: persistence of opportunistic fungal pathogens in the human host. Curr. Opin. Microbiol, 12, 358-364 (2009).

2. Lu, M. et al. Antifungal effects of phytocompounds on Candida species alone and in combination with fluconazole. Int. J. Antimicrob. Agents, 49, 125-136 (2009).

3. Brown, G. D. et al. Hidden killers: human fungal infections. SciTransl Med. 4, 165rv13-165rv13(2012).

4. Chakraborti, S., Ramakrishnan, G. \& Srinivasan, N. In Silico Modeling of FDA-Approved Drugs for Discovery of Anticandida Agents: A Drug-Repurposing Approach. (Ed. Roy, K.) In Silico Drug Design: Repurposing Techniques and Methodologies. Academic Press. 463-526(2019).

5. Roscetto, E. et al. Antifungal and anti-biofilm activity of the first cryptic antimicrobial peptide from an archaeal protein against Candida spp. clinical isolates. Sci. Rep, 8, 1-11 (2018).

6. Jang, S. J., Lee, K., Kwon, B., You, H. J. \& Ko, G. Vaginal lactobacilli inhibit growth and hyphae formation of Candida albicans. Scientific reports. 9.1-9(2019).

7. Kornitzer, D. Regulation of Candida albicans hyphal morphogenesis by endogenous signals. J.Fungi, 5, 21 (2019).

8. Leite, M. C. A., de Brito Bezerra, A. P., de Sousa, J. P. \& de Oliveira Lima, E. Investigating the antifungal activity and mechanism (s) of geraniol against Candida albicans strains. Med. Mycol, 53, 275-284 (2015).

9. Calderone, R. A. \& Fonzi, W. A. Virulence factors of Candida albicans. Trends Microbiol.327-335(2001).

10. Jacobsen, I. D. et al. Candida albicans dimorphism as a therapeutic target. Expert review of anti-infective therapy, 10, 85-93 (2012).

11. Chin, V. K., Lee, T. Y., Rusliza, B. \& Chong, P. P. Dissecting Candida albicans infection from the perspective of C. albicans virulence and omics approaches on host-pathogen interaction: a review. Int. J. Mol. Sci. 17, 1643 (2016).

12. Lo, H. J. et al.. albicans mutants are avirulent. Cell, 90, 939-949 (1997).

13. Gale, C. A. et al. 1998. Linkage of adhesion, filamentous growth, and virulence in Candida albicans to a single gene, INT1. Science 279(5355), 1355-1358 (1998).

14. Han, T. L., Cannon, R. D. \& Villas-Bôas, S. G. The metabolic basis of Candida albicans morphogenesis and quorum sensing. Fungal Genet Biol, 48, 747-763 (2011). 
15. Ksiezopolska, E. \& Gabaldón, T. Evolutionary emergence of drug resistance in Candida opportunistic pathogens. Genes, 9, 461 (2018).

16. Gauwerky, K., Borelli, C. \& Korting, H. C. Targeting virulence: a new paradigm for antifungals. Drug discovery today, 14, 214-222 (2009).

17. Cui, J., Ren, B., Tong, Y., Dai, H. \& Zhang, L. Synergistic combinations of antifungals and anti-virulence agents to fight against Candida albicans. Virulence, 6, 362-371 (2015).

18. Kocaçalışkan, I., Talan, I. \& Terzi, I. Antimicrobial activity of catechol and pyrogallol as allelochemicals. $Z$ Naturforsch C J Biosci, 61, 639-642 (2006).

19. Vishakha, K., Das, S., Banerjee, S., Mondal, S. \& Ganguli, A. Allelochemical catechol comprehensively impedes bacterial blight of rice caused by Xanthomonasoryzaepv. oryzae. Microb. Pathogen, 149, 104559 (2020).

20. Farkas, G. L. \& Kiraaly, Z. Role of phenolic compounds in the physiology of plant diseases and disease resistance. J. Phytopathol, 44, 105-150 (1962).

21. González-López, J. A., Pérez-Mondragón, A. A., Cuevas-Suárez, C. E., Trejo-Carbajal, N. \& Herrera-González, A. M. Evaluation of dental composites resins formulated with non-toxic monomers derived from catechol. J. Mech. Behav. Biomed. Mater, 104, 103613 (2020).

22. Ryu, J. H. et al. Catechol-functionalized chitosan/pluronic hydrogels for tissue adhesives and hemostatic materials. Biomacromolecules.12. 2653-2659(2011).

23. Berlanga Duarte, M. L., Reyna Medina, L. A., Reyes, P. T., Gonzalez Perez, S. E. \& Herrera González, A. M. Biobased isosorbide methacrylate monomer as an alternative to bisphenol A glycerolatedimethacrylate for dental restorative applications.J. Appl. Polym. Sci.134 (2017).

24. Kim, K. H. et al. (Tandem conversion of lignin to catechols via demethylation and catalytic hydrogenolysis. Industrial Crops and Products2021).

25. FarisaBanu, S., Thamotharan, S., Gowrishankar, S., Karutha Pandian, S. \& Nithyanand, P. Marine bacterial DNase curtails virulence and disrupts biofilms of Candida albicans and non-albicans Candida species. Biofouling, 35, 975-985 (2019).

26. Repp, K. K., Menor, S. A. \& Pettit, R. K. Microplate Alamar blue assay for susceptibility testing of Candida albicans biofilms. Med. Mycol, 45, 603-607 (2007).

27. Prasath, K. G., Sethupathy, S. \& Pandian, S. K. Proteomic analysis uncovers the modulation of ergosterol, sphingolipid and oxidative stress pathway by myristic acid impeding biofilm and virulence in Candida albicans. J. Proteomics, 208, 103503 (2019).

28. Gowrishankar, S. \& Pandian, S. K. Modulation of Staphylococcus epidermidis (RP62A) extracellular polymeric layer by marine cyclic dipeptide-cyclo (I-leucyl-I-prolyl) thwarts biofilm formation. Biochimica et BiophysicaActa (BBA)-Biomembranes. 1859, 1254-1262(2017).

29. Bar-Yosef, H., Gonzalez, N. V., Ben-Aroya, S., Kron, S. J. \& Kornitzer, D. Chemical inhibitors of Candida albicans hyphal morphogenesis target endocytosis. Sci. Rep, 7, 1-12 (2017).

30. Lee, J. H. et al. Antibiofilm and antivirulence activities of 6-gingerol and 6-shogaol against Candida albicans due to hyphal inhibition. Front. Cell. Infect. Microbiol, 8, 299 (2018).

31. Muthamil, S., Balasubramaniam, B., Balamurugan, K. \& Pandian, S. K. Synergistic effect of quinic acid derived from Syzygiumcumini and undecanoic acid against Candida spp. biofilm and virulence. Front. 
Microbiol, 9, 2835 (2018).

32. Akçağlar, S., Ener, B. \& Töre, O. Acid proteinase enzyme activity in Candida albicans strains: a comparison of spectrophotometry and plate methods. Turk. J. Biol, 35, 559-567 (2011).

33. Silva-Dias, A. et al. Adhesion, biofilm formation, cell surface hydrophobicity, and antifungal planktonic susceptibility: relationship among Candida spp.Frontiers in microbiology.6(2015).

34. Priya, A. \& Pandian, S. K. Piperine impedes biofilm formation and hyphal morphogenesis of Candida albicans. Front. Microbiol.11 (2020).

35. Abirami, G., Alexpandi, R., Durgadevi, R., Kannappan, A. \& Ravi, A. V. Inhibitory effect of morin against Candida albicans pathogenicity and virulence factor production: An in vitro and in vivo approaches. Front. Microbiology. 11 (2020).

36. Cavalheiro, M. \& Teixeira, M. C. Candida biofilms: threats, challenges, and promising strategies. Front. Med, 5, 28 (2018).

37. Badireddy, A. R. et al. Role of extracellular polymeric substances in bioflocculation of activated sludge microorganisms under glucose-controlled conditions. Water. Res, 44, 4505-4516 (2010).

38. Arthington-Skaggs, B. A., Jradi, H., Desai, T. \& Morrison, C. J. Quantitation of ergosterol content: novel method for determination of fluconazole susceptibility of Candida albicans. J. Clin. Microbiol, 37, 33323337 (1999).

39. Hornby, J. M. \& Nickerson, K. W. Enhanced production of farnesol by Candida albicans treated with four azoles. Antimicrob. Agents Chemother, 48, 2305-2307 (2004).

40. Pierce, C. G. et al. A novel small molecule inhibitor of Candida albicans biofilm formation, filamentation and virulence with low potential for the development of resistance. npj Biofilms and Microbiomes, 1, 1-8 (2015).

41. Bachmann, S. P. et al. Antifungal combinations against Candida albicans biofilms in vitro. Antimicrob. Agents Chemother, 47, 3657-3659 (2003).

42. de Oliveira Santos, G. C. et al. do ; ; ; and de Andrade Monteiro, C. Candida infections and therapeutic strategies: mechanisms of action for traditional and alternative agents. Front. Microbiol. 9, 1351(2018).

43. Mayer, F. L. ; Duncan Wilson, and Bernhard Hube. Candida albicans.119-128.

44. Saville, S. P., Lazzell, A. L., Monteagudo, C. \& Lopez-Ribot, J. L. Engineered control of cell morphology in vivo reveals distinct roles for yeast and filamentous forms of Candida albicans during infection. Eukaryot. Cell, 2, 1053-1060 (2003).

45. Pandey, N., Gupta, M. K. \& Tilak, R. Extracellular hydrolytic enzyme activities of the different Candida spp. isolated from the blood of the Intensive Care Unit-admitted patients. J Lab Physicians, 10, 392 (2018).

46. Hoeg, L., Thoma-Greber, E., Röcken, M. \& Korting, H. C. HIV protease inhibitors influence the prevalence of oral candidosis in HIV-infected patients: a 2-year study: HIV-Protease-Inhibitorenbeeinflussen die HäufigkeitoralerCandidosebei HIV-Patienten. EineZweijahresstudie. Mycoses, 41, 321-325 (1998).

47. Ganendren, R. et al. In vitro antifungal activities of inhibitors of phospholipases from the fungal pathogen Cryptococcus neoformans. Antimicrob. Agents Chemother, 48, 1561-1569 (2004).

48. Krasowska, A. \& Sigler, K. How microorganisms use hydrophobicity and what does this mean for human needs? Front. Cell. Infect. Microbiol, 4, 112 (2014). 
49. Chandra, J. et al. Biofilm formation by the fungal pathogen Candida albicans: development, architecture, and drug resistance. J. Bacteriol, 183, 5385-5394 (2001).

50. Sivaranjani, M. et al. Morin inhibits biofilm production and reduces the virulence of Listeria monocytogenes -An in vitro and in vivo approach. Int. J. Food Microbiol, 237, 73-82 (2016).

51. Flemming, H. C. \& Wingender, J. Relevance of microbial extracellular polymeric substances (EPSs)-Part I: Structural and ecological aspects. Water Sci. Technol, 43, 1-8 (2001).

52. Bhattacharya, S., Sae-Tia, S. \& Fries, B. C. Candidiasis and mechanisms of antifungal resistance.J. Antibiot.312(2020).

53. Rajasekharan, S. K. et al. Mannich base limits Candida albicans virulence by inactivating Ras-cAMP-PKA pathway. Sci. Rep, 8, 1-9 (2018).

54. Lu, Y., Su, C. \& Liu, H. Candida albicans hyphal initiation and elongation. Trends Microbiol, 22, 707-714 (2014).

55. Li, Y. et al. S. The synergistic antifungal effect and potential mechanism of D-penicillamine combined with fluconazole against Candida albicans. Front. Microbiol, 10, 2853 (2019).

56. Khan, Z., Ahmad, S., Joseph, L. \& Chandy, R. Candida dubliniensis: an appraisal of its clinical significance as a bloodstream pathogen. PLoS One, 7, 32952 (2012).

57. Shinde, R. B., Raut, J. S. \& Karuppayil, M. S. Biofilm formation by Candida albicans on various prosthetic materials and its fluconazole sensitivity: a kinetic study. Mycoscience, 53, 220-226 (2012).

58. Kebaara, B. W. et al. Candida albicans Tup1 is involved in farnesol-mediated inhibition of filamentousgrowth induction. Eukaryotic cell, 7 (6), 980-987 (2008).

\section{Table}

Table 1. List of the genes, their function and corresponding primer sequence used in the current study. 


\begin{tabular}{|c|c|c|c|c|}
\hline \multirow{2}{*}{$\begin{array}{l}\text { S. } \\
\text { No }\end{array}$} & \multirow[t]{2}{*}{ Gene } & \multirow[t]{2}{*}{ Function } & \multicolumn{2}{|l|}{ Primer sequence $\left(5^{\prime}-3^{\prime}\right)$} \\
\hline & & & Forward & Reverse \\
\hline 1 & $n r g 1$ & $\begin{array}{l}\text { Negative } \\
\text { regulator of } \\
\text { transcription }\end{array}$ & CCAAGTACCTCCACCAGCAT & GGGAGTTGGCCAGTAAATCA \\
\hline 2 & tup 1 & $\begin{array}{l}\text { Negative } \\
\text { regulator of } \\
\text { transcription }\end{array}$ & CTTGGAGTTGGCCCATAGAA & TGGTGCCACAATCTGTTGTT \\
\hline \multirow[t]{2}{*}{3} & hwp 1 & $\begin{array}{l}\text { Hyphal wall } \\
\text { protein }\end{array}$ & GCTCCTGCTCCTGAAATGAC & CTGGAGCAATTGGTGAGGTT \\
\hline & & Adhesin & & \\
\hline 4 & ras1 & $\begin{array}{l}\text { Cell } \\
\text { adhesion, } \\
\text { filamentous } \\
\text { growth }\end{array}$ & CCCAACTATTGAGGATTCTTATCGTAAA & TCTCATGGCCAGATATTCTTCTTG \\
\hline 5 & als3 & $\begin{array}{l}\text { Adhesion, } \\
\text { Agglutinin } \\
\text { like protein }\end{array}$ & CAACTTGGGTTATTGAAACAAAAACA & AGAAACAGAAACCCAAGAACAACC \\
\hline
\end{tabular}

\section{Figures}

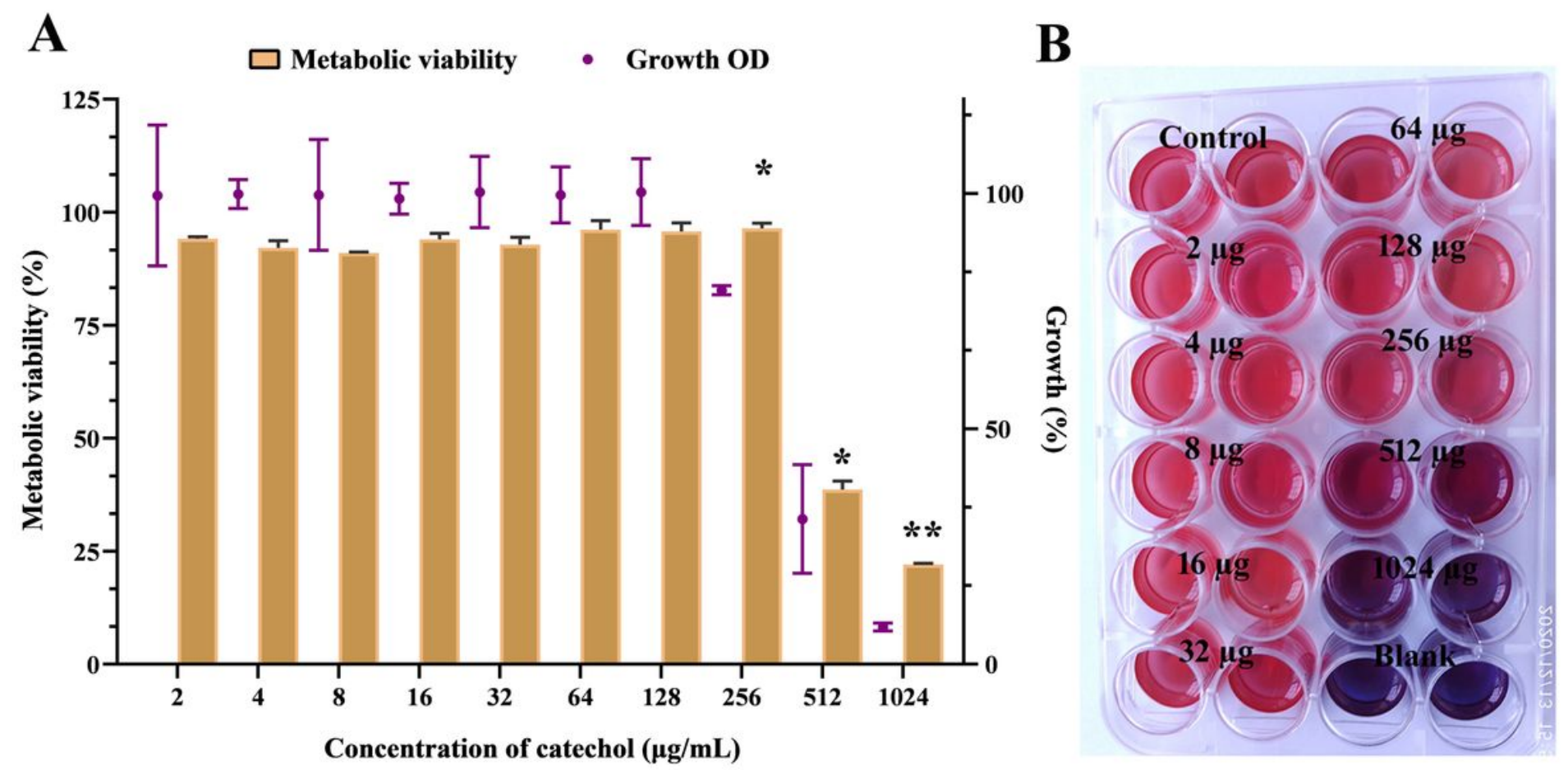

\section{Figure 1}

The influence of catechol (0-1024 $\mu \mathrm{g} / \mathrm{mL})$ on C. albicans planktonic growth. (A) Measurement of C. albicans cell density and metabolic viability after grown for $24 \mathrm{~h}$ in the presence and absence of catechol using broth micro dilution and alamar blue assay, respectively. Error bars indicates the mean values of three experimental 


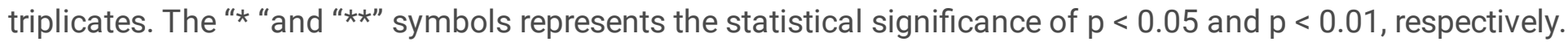
(B) Representative MTP stained with alamar blue show the true metabolic state of catechol treated C. albicans cells.

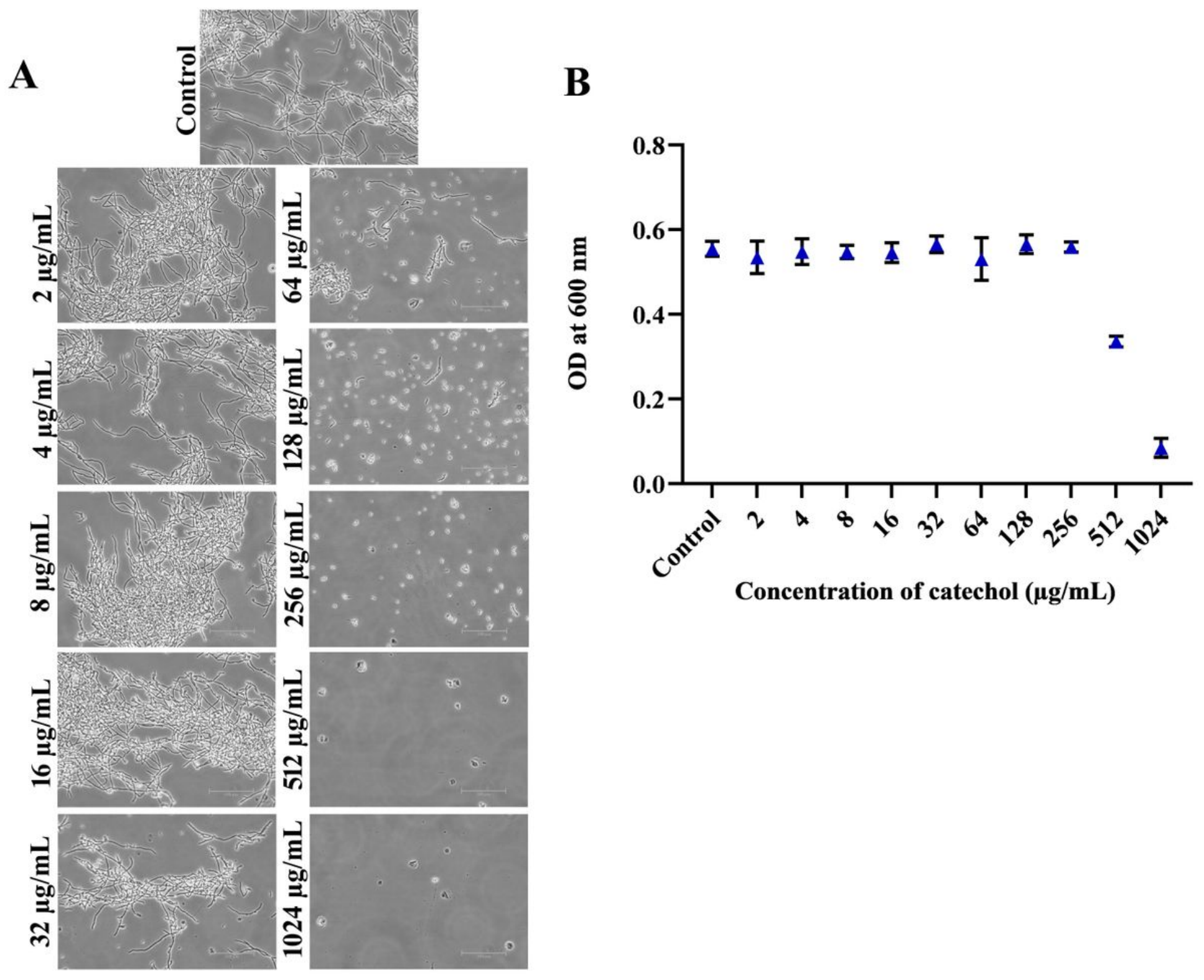

Figure 2

Effect of catechol on yeast to hyphae transition and planktonic growth. (A) C. albicans was grown on liquid spider medium with and without catechol. After $24 \mathrm{~h}$ incubation, test samples were photographed under phase contrast microscope. Micrograph of control group exposed very dense and lengthy filamentous cells, catechol treated groups exposed a more number of evenly distributed yeast cells. (B) Impact of catechol at various concentrations on C. albicans planktonic growth in spider broth after $24 \mathrm{~h}$ incubation. 
Control

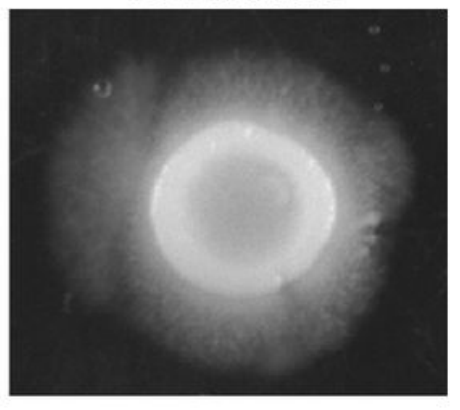

$64 \mu \mathrm{g} / \mathrm{mL}$

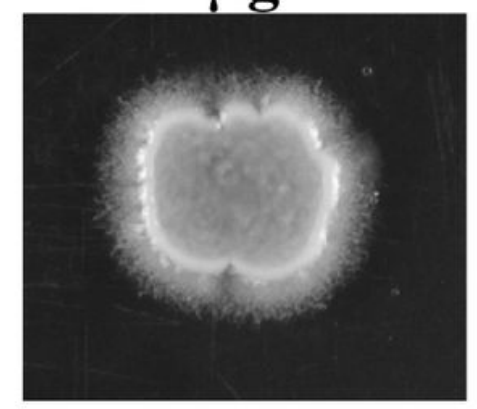

$128 \mu \mathrm{g} / \mathrm{mL}$

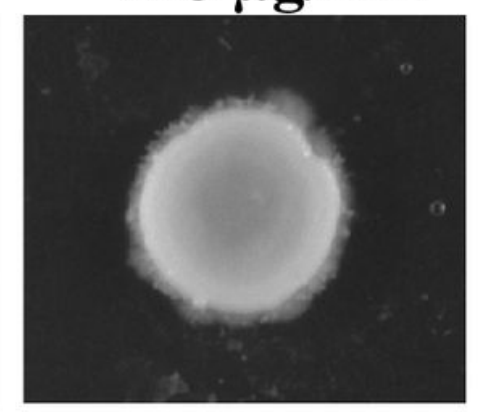

$256 \mu \mathrm{g} / \mathrm{mL}$

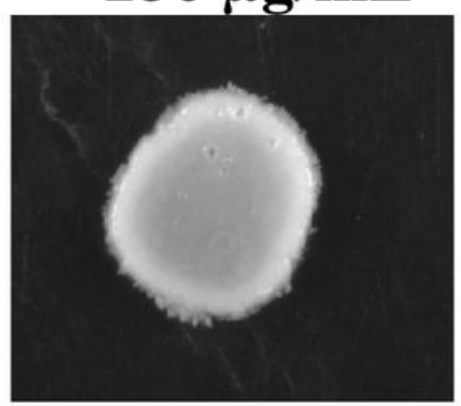

\section{Figure 3}

Conformation of yeast to hyphae inhibition by catechol on solid spider media. The fungal colonies were imaged after five days of incubation at $37^{\circ} \mathrm{C}$. Colonies without catechol were bare the deep hyphal protrusion with irregular cell morphology whereas, colonies treated with catechol displayed a non-filamentous and very smooth topography. 

A
Biofilm cells
- Planktonic cells
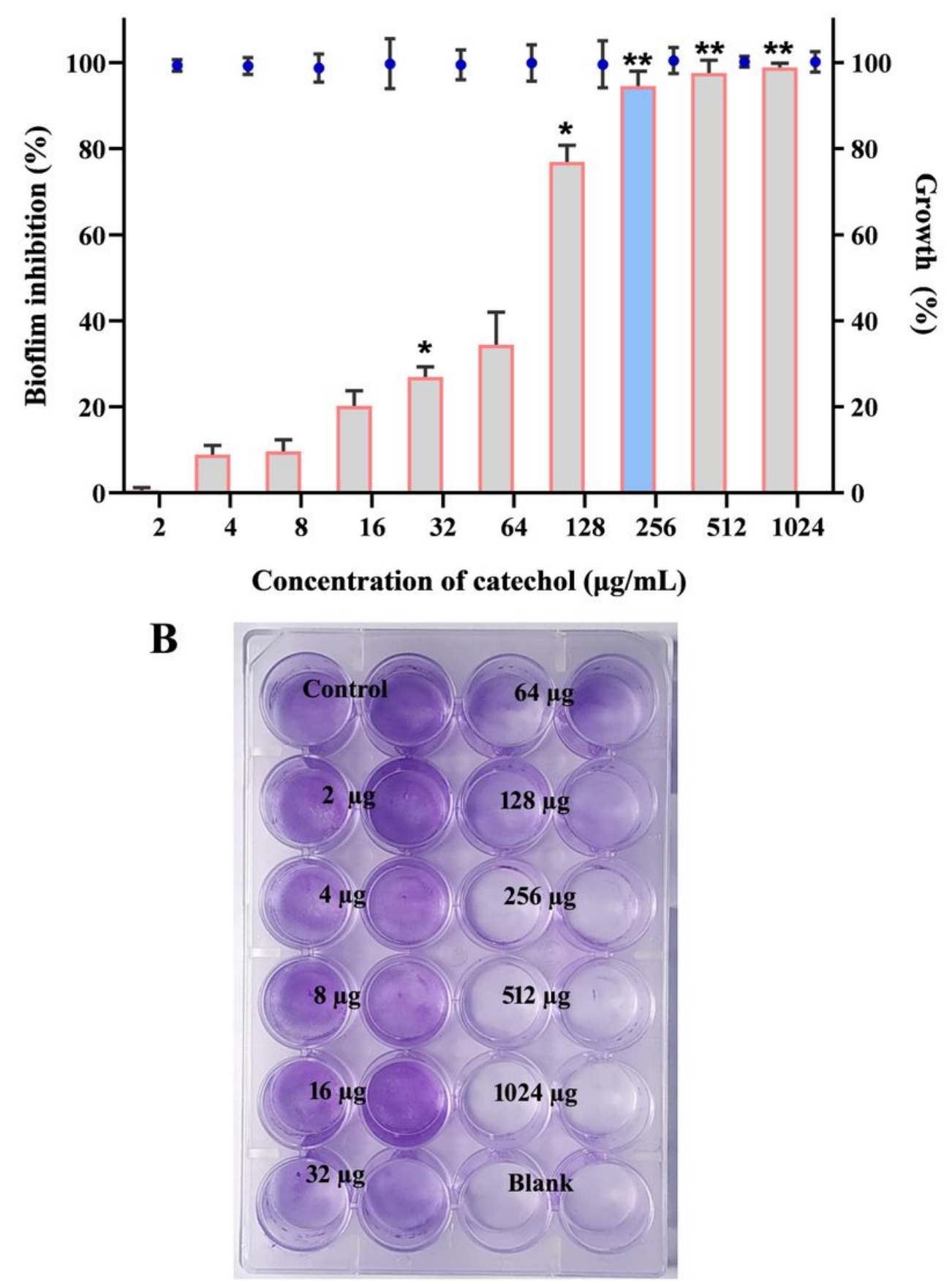

\section{Figure 4}

Antibiofilm efficacy of catechol at varied concentrations. (A) Measurement of biofilm formation and planktonic growth in presence of catechol at various concentrations. Significant dose dependent reductions of biofilm formation were observed in the cells treated with catechol compared to untreated control without affecting the C. albicans planktonic growth. Blue colored bar in the graph signifies the BIC value of catechol. Error bars indicates the mean values of three experimental triplicates. The "* "and " $\star \star$ " symbols represents the statistical significance of $p<0.05$ and $p<0.01$, respectively. (B) Representative MTP image that portraying the impact of catechol on C. albicans biofilm. 


\section{Control}

A

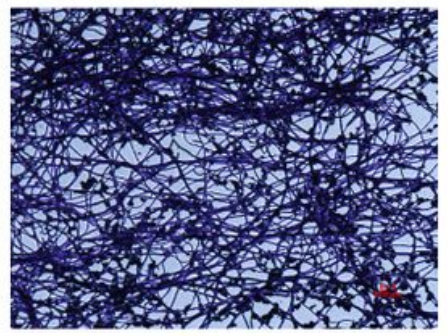

B

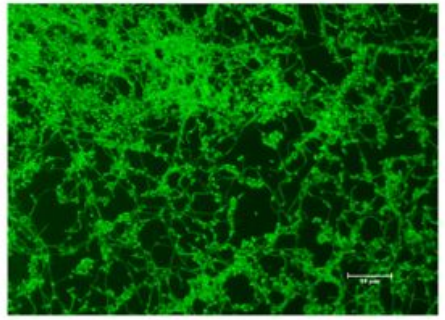

$64 \mu \mathrm{g} / \mathrm{mL}$
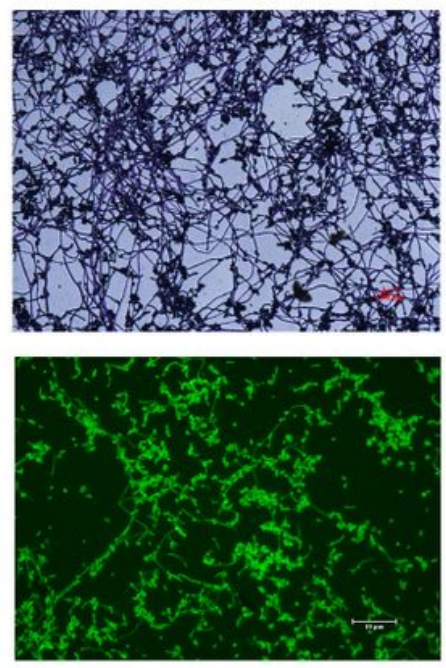

$128 \mu \mathrm{g} / \mathrm{mL}$
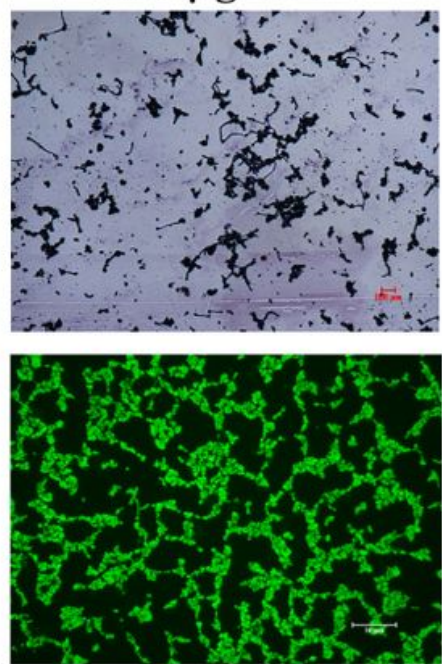

$256 \mu \mathrm{g} / \mathrm{mL}$
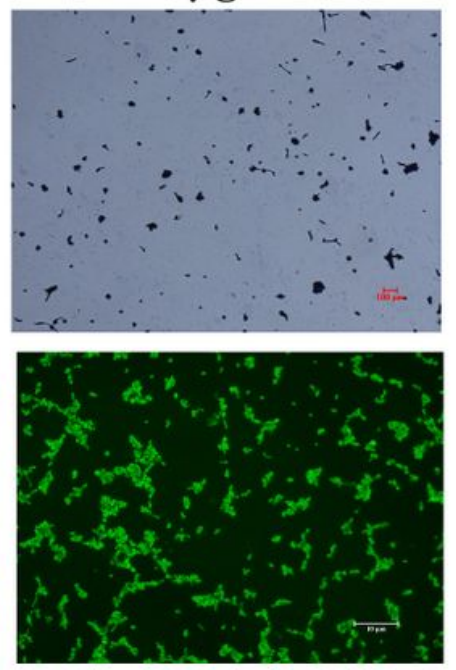

Figure 5

Microscopic visualization of C. albicans biofilms formed on the glass surface after $48 \mathrm{~h}$ of incubation at $37^{\circ} \mathrm{C}$ and subsequent staining with $0.1 \%$ acridine orange or $0.4 \%$ of crystal violet. (A) Light ( $200 \mathrm{X}$ magnification) (B) Fluorescence micrograph of control samples showed tightly packed biofilms architectures surrounded with both yeast and hyphal cells. While, the micrograph of catechol treated samples depicted that the considerable decrease in the hyphal and biofilm development. (Magnification: $200 \mathrm{X}$, Scale bar: $50 \mu \mathrm{m}$ or $100 \mu \mathrm{m}$ ).
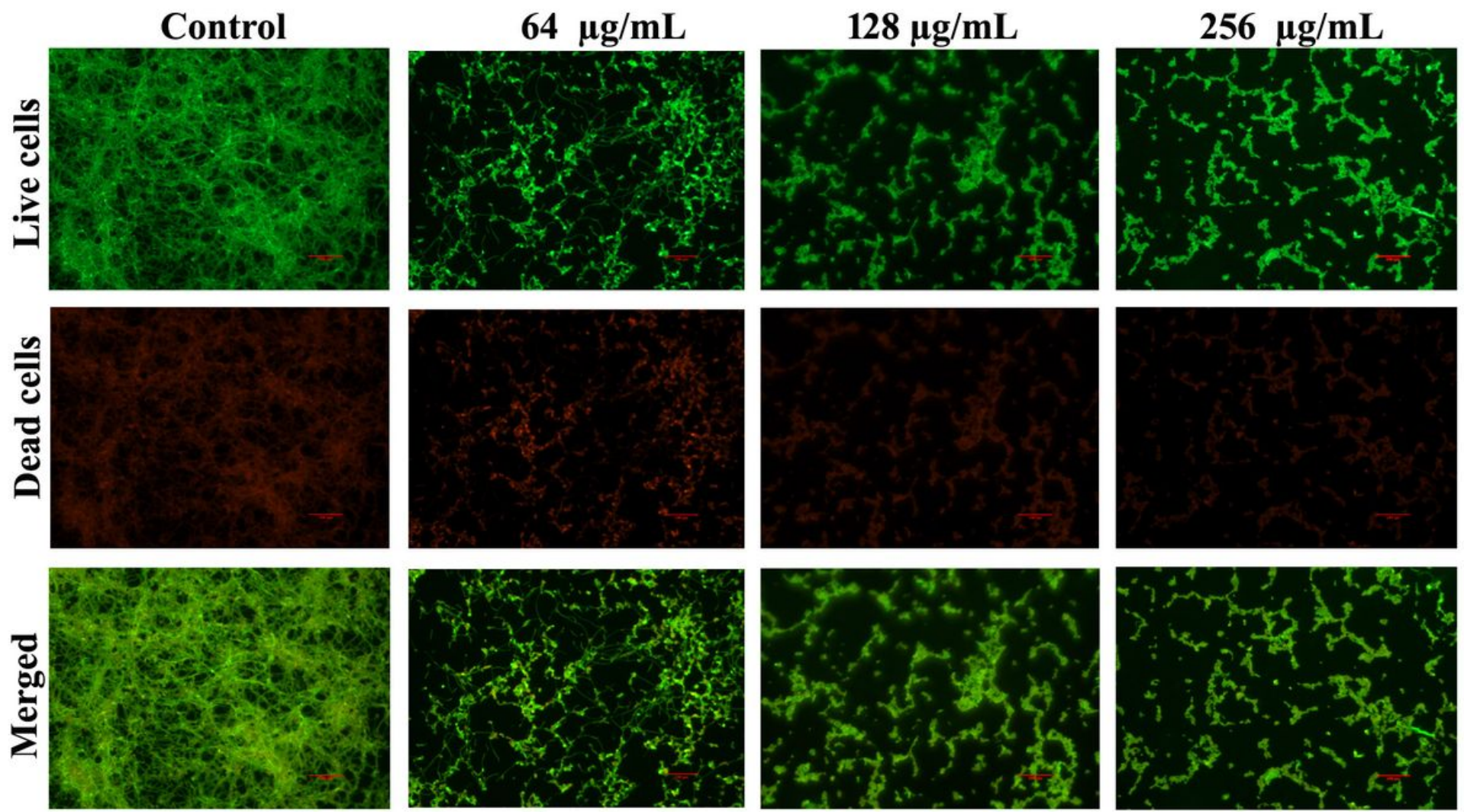

Figure 6 
LIVE/DEAD analysis of $48 \mathrm{~h}$ catechol treated and untreated C. albicans sessile cells. The micrographic images of both catechol treated and untreated control samples reveals the presence of more number of live cells compared to dead cells. Magnification: 200 X, Scale bar: $50 \mu \mathrm{m})$.

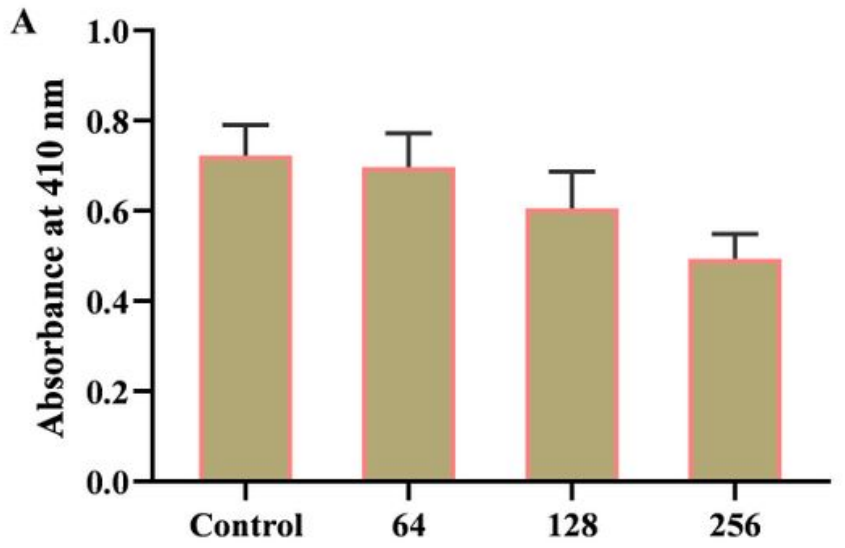

B

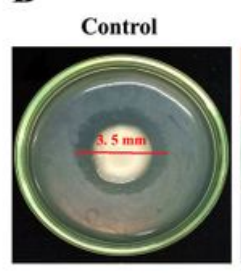

Concentration of catechol $(\mu \mathrm{g} / \mathrm{mL})$

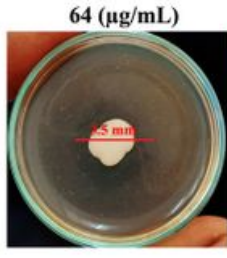

$128(\mu \mathrm{g} / \mathrm{mL})$

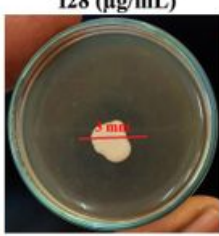

C

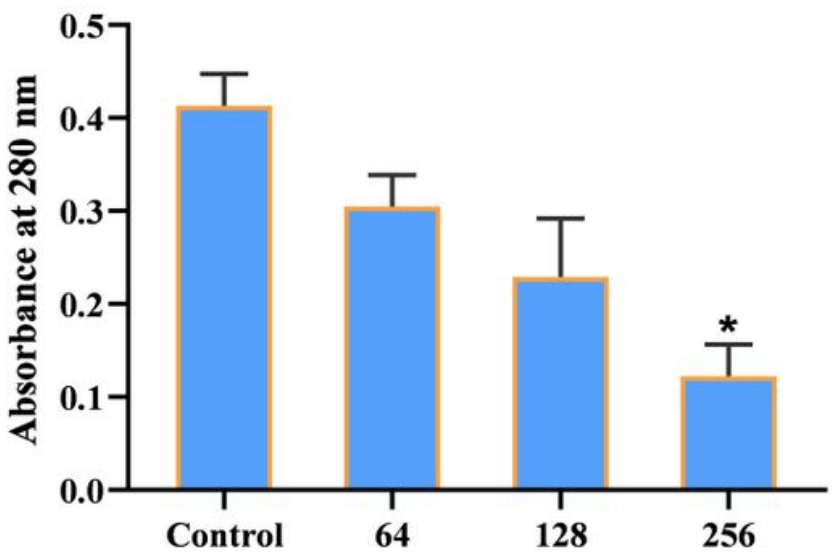

D

Concentration of catechol $(\mu \mathrm{g} / \mathrm{mL})$

$256(\mu \mathrm{g} / \mathrm{mL})$
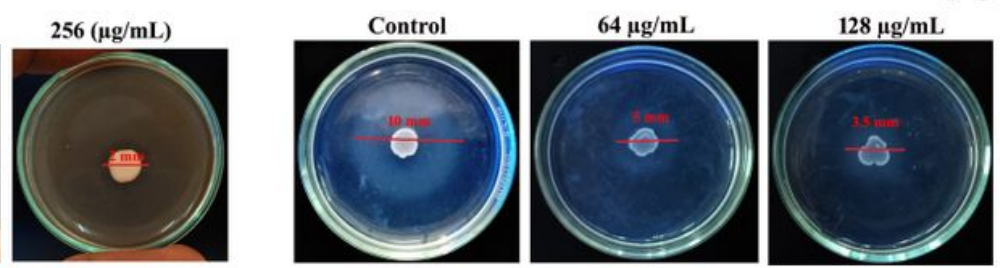

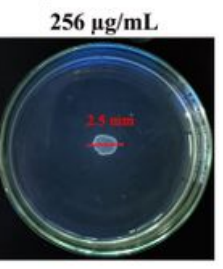

\section{Figure 7}

Impact of catechol on virulence enzyme production. (A) Quantification of C. albicans lipase production after 24 $\mathrm{h}$ treatment with catechol at the concentration of 64,128 and $256 \mu \mathrm{g} / \mathrm{mL}$. Error bars indicates the mean values of two experimental triplicates. (B) C. albicans culture was spotted on the plate supplemented with tributyrin in presence and absence of catechol to detect the lipase activity. (C) Quantification of C. albicans protease production after $24 \mathrm{~h}$ treatment with and without catechol (at 64, 128 and $256 \mu \mathrm{g} / \mathrm{mL}$ ) using BSA as a substrate. Error bars indicates the two experiments triplicates.. (D) Qualitative assessment of catechol inhibitory efficacy on the $\mathrm{C}$. albicans protease production using BSA plate. Error bars indicates the mean values of two experiments triplicates. The "* "and “**” symbols represents the statistical significance of $p<0.05$ and $p<0.01$, respectively. 


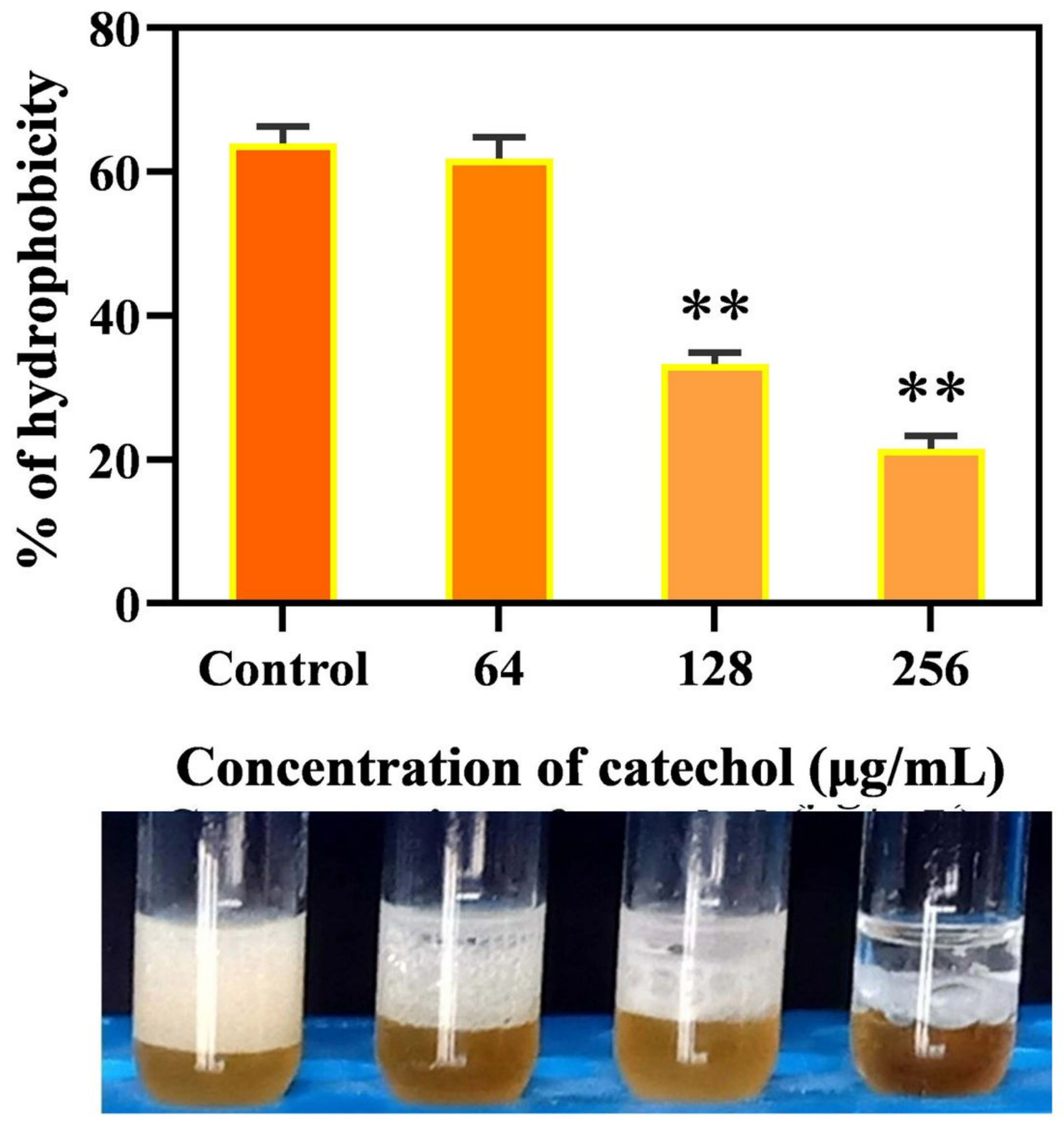

Figure 8

Effect of catechol at its sub-MIC on the C. albicans cellular surface hydrophobicity after grown for $24 \mathrm{~h}$. Error

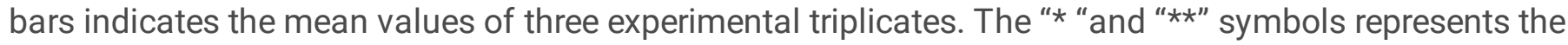
statistical significance of $p<0.05$ and $p<0.01$, respectively. 


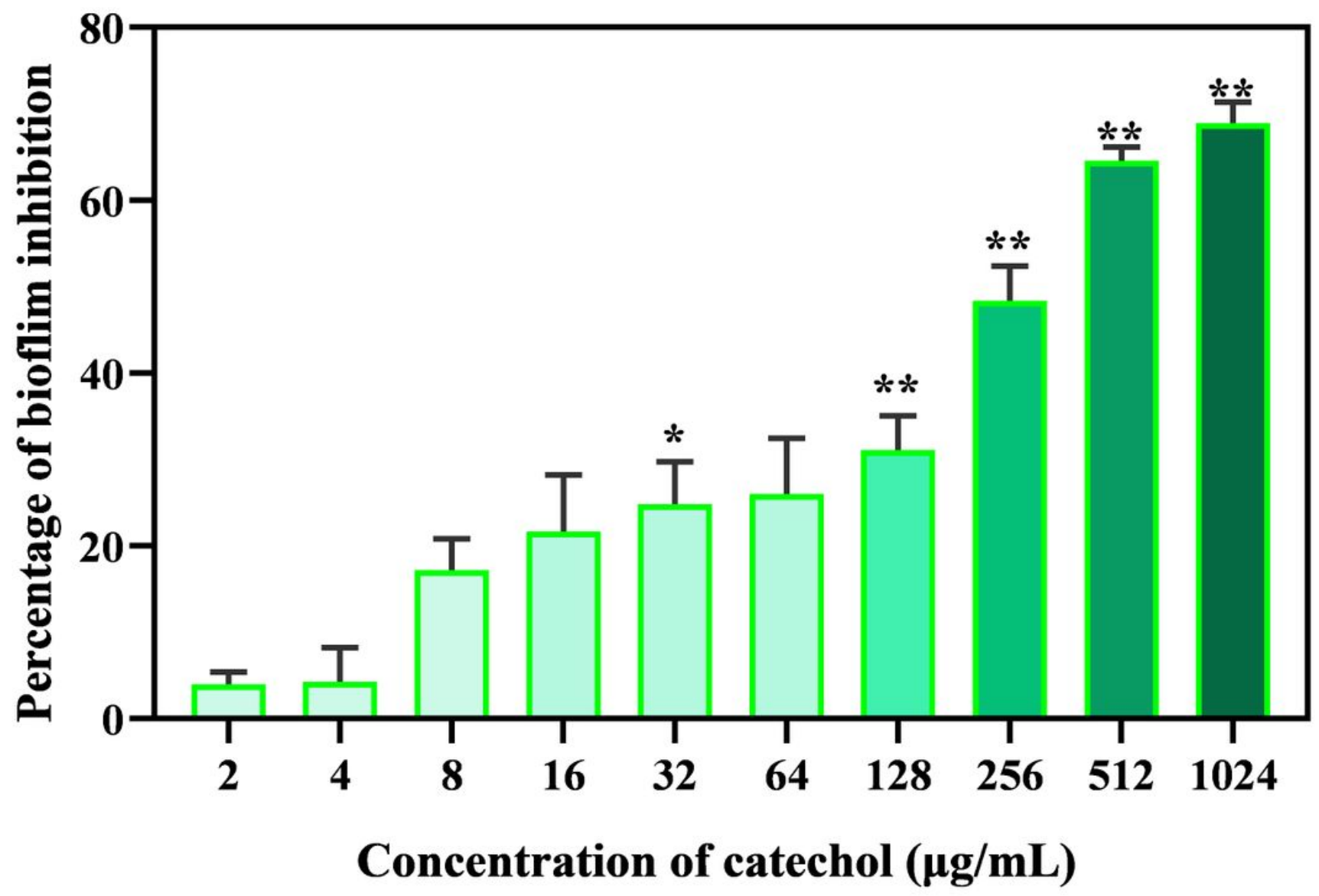

Figure 9

The evaluation of catechol efficacy on preformed biofilm of $\mathrm{C}$. albicans. Error bars indicates the mean values of

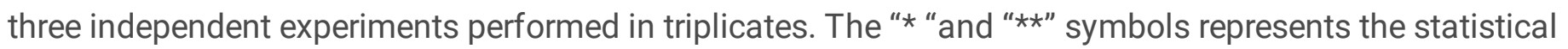
significance of $p<0.05$ and $p<0.01$, respectively. 


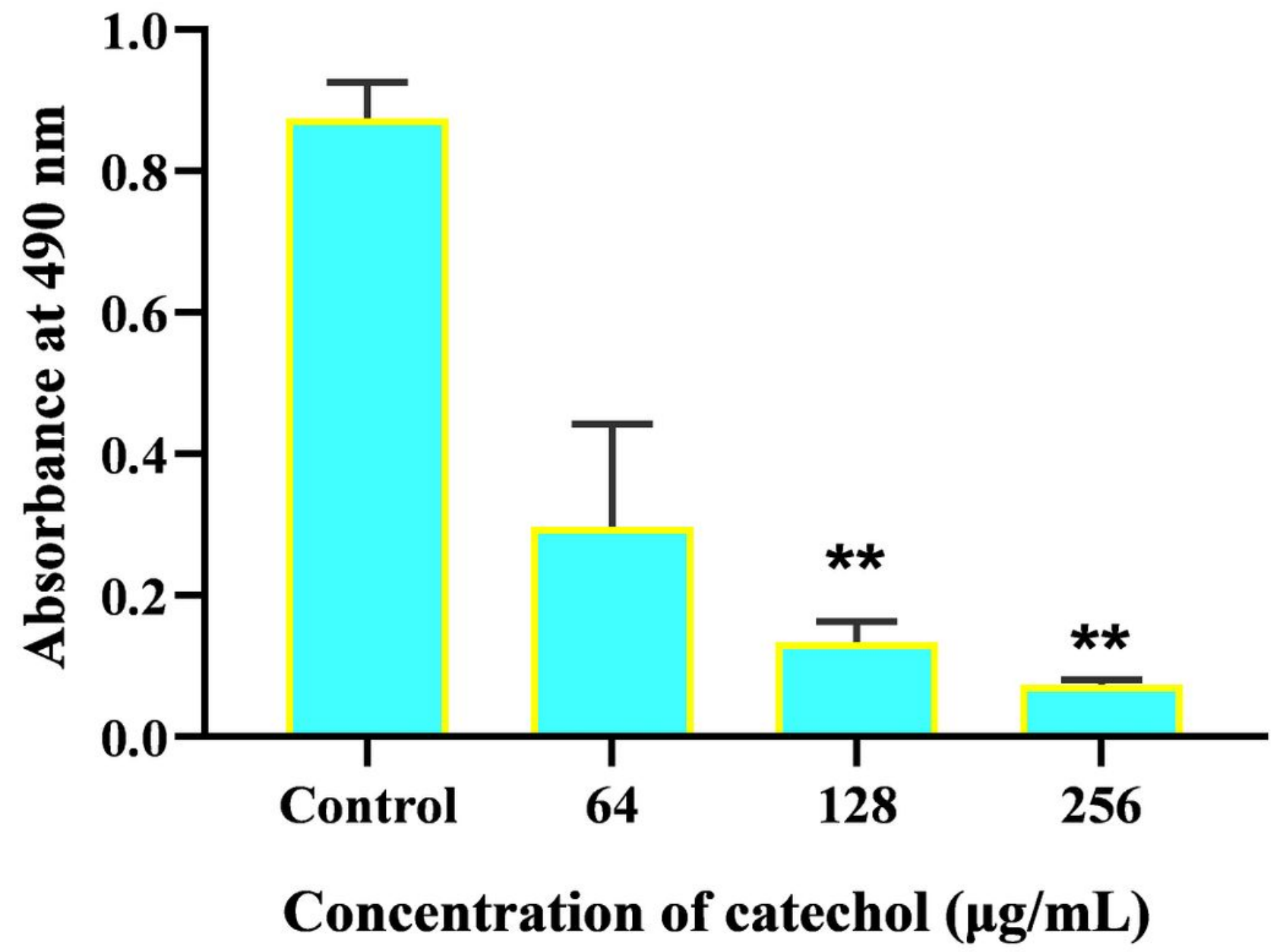

Figure 10

Quantification of total polysaccharide content in C. albicans after grown $24 \mathrm{~h}$ with and without catechol using phenol- suphuric acid method. Error bars indicates the mean values of two experimental triplicates. The " "and

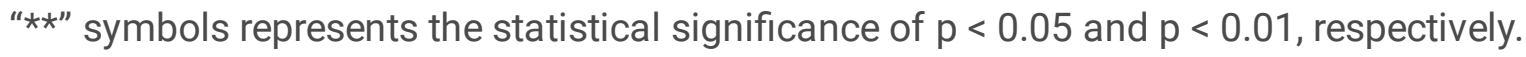




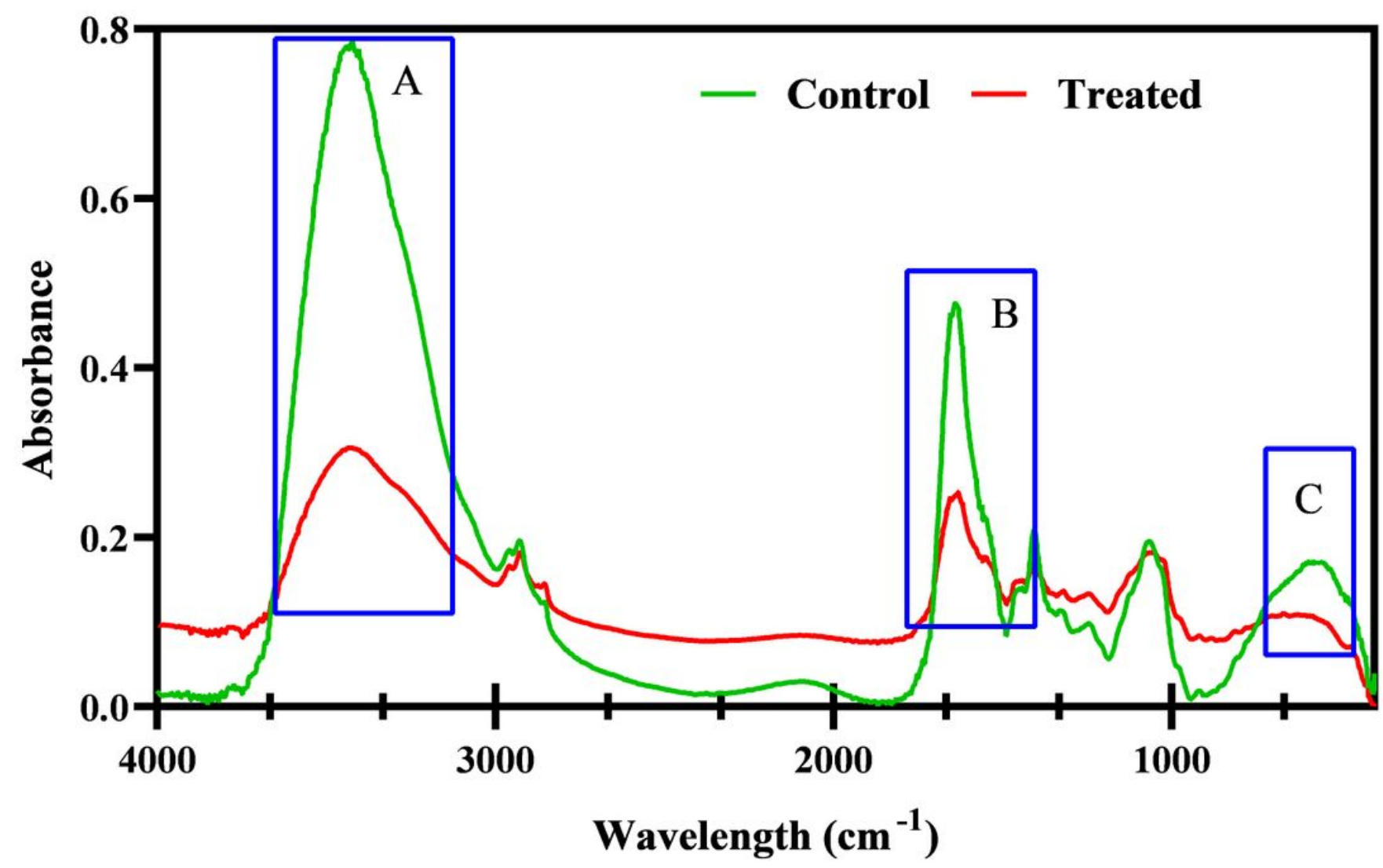

Figure 11

FTIR analysis of C. albicans EPS. Spectral region ranging from 4000-400 cm-1 represent the control and treated samples. Highlighted region portray the decrease in catechol treated EPS component such as amide bonds of peptides (3200- $3800 \mathrm{~cm}-1)$, proteins (1500- $1800 \mathrm{~cm}-1)$ and polysaccharides $(200-600 \mathrm{~cm}-1)$ of

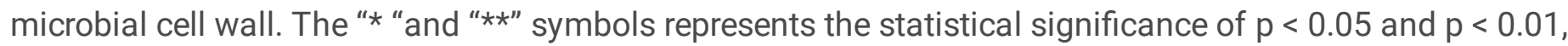
respectively. 


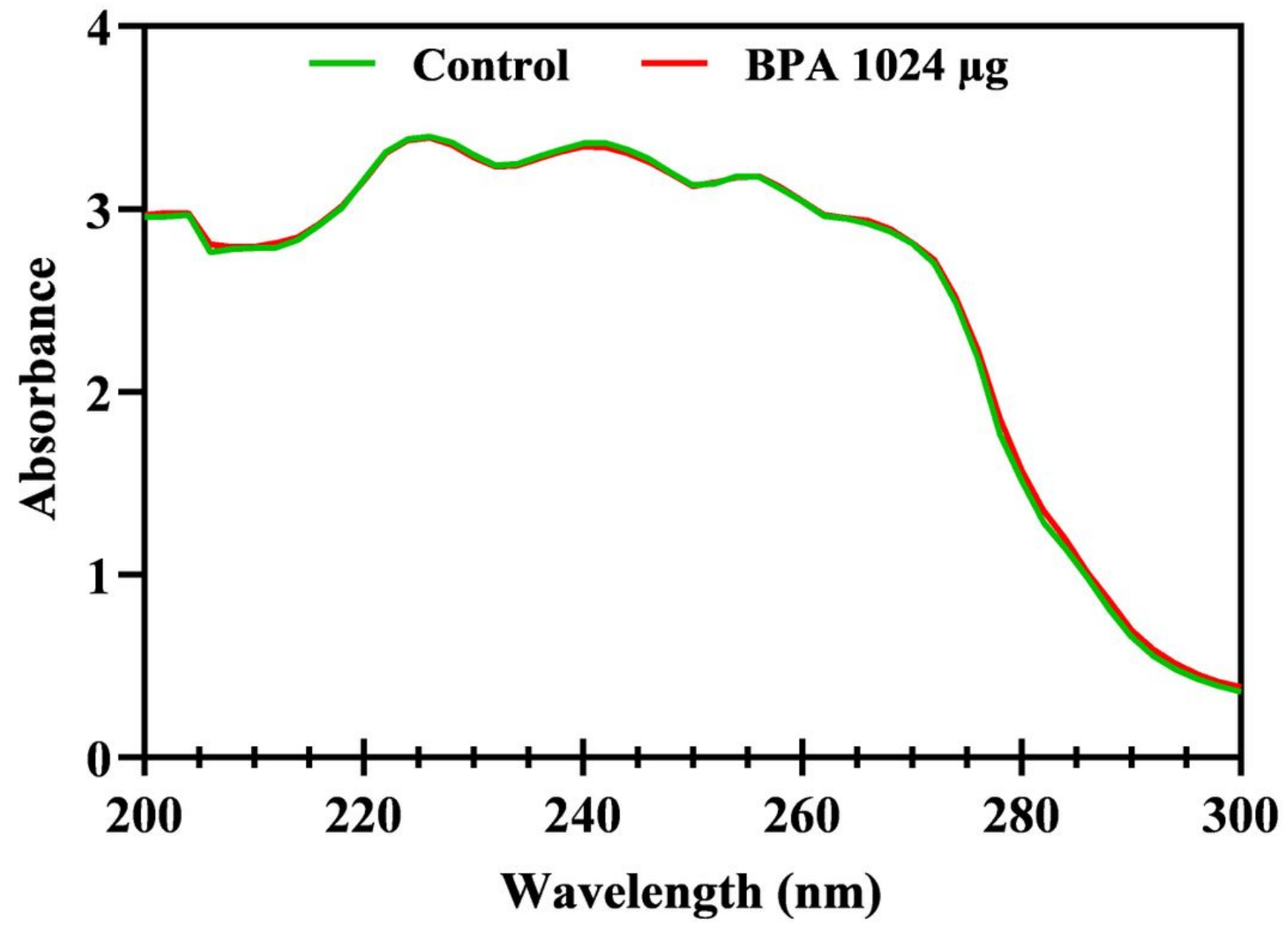

Figure 12

No, influence of catechol (at $256 \mu \mathrm{g} / \mathrm{mL}$ ) on C. albicans ergosterol production after $24 \mathrm{~h}$ treatment. 


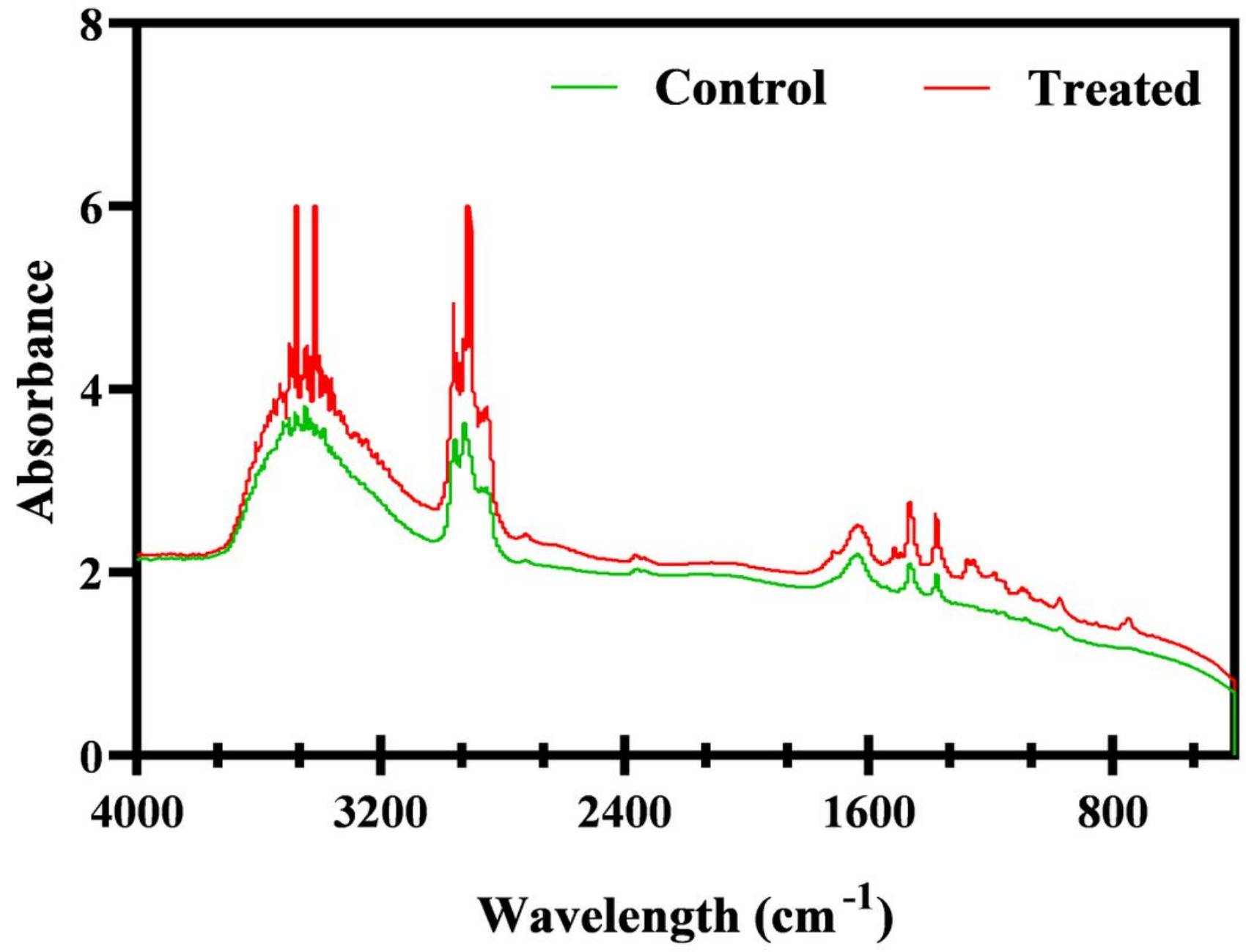

Figure 13

FTIR analysis of C. albicans farnesol upon $24 \mathrm{~h}$ treatment with catechol (at $256 \mu \mathrm{g} / \mathrm{mL}$ ). 
A
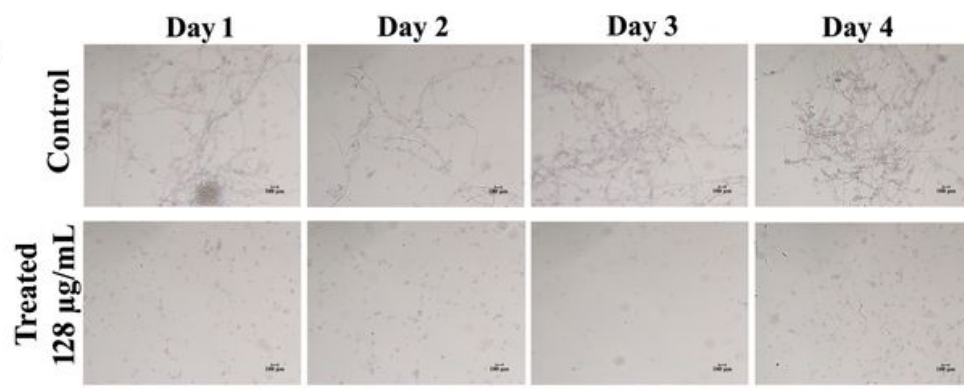

Day 4

Day 5

Day 6

Day 7

Day 8
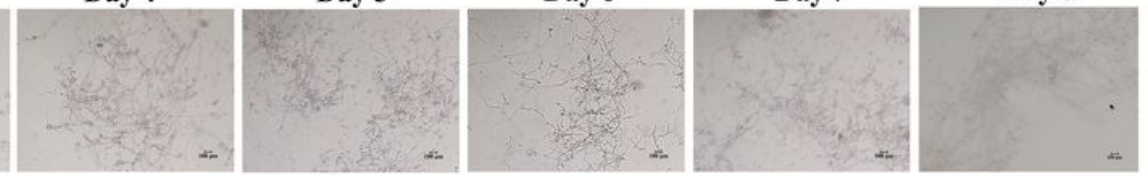

Day 9

Day 10

Day 11

Day 12
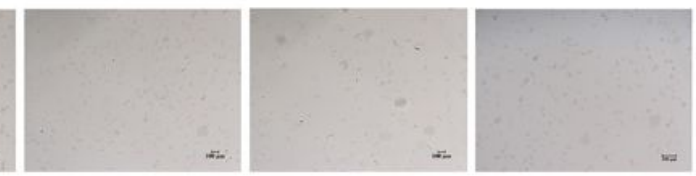

Day 14

Day 15
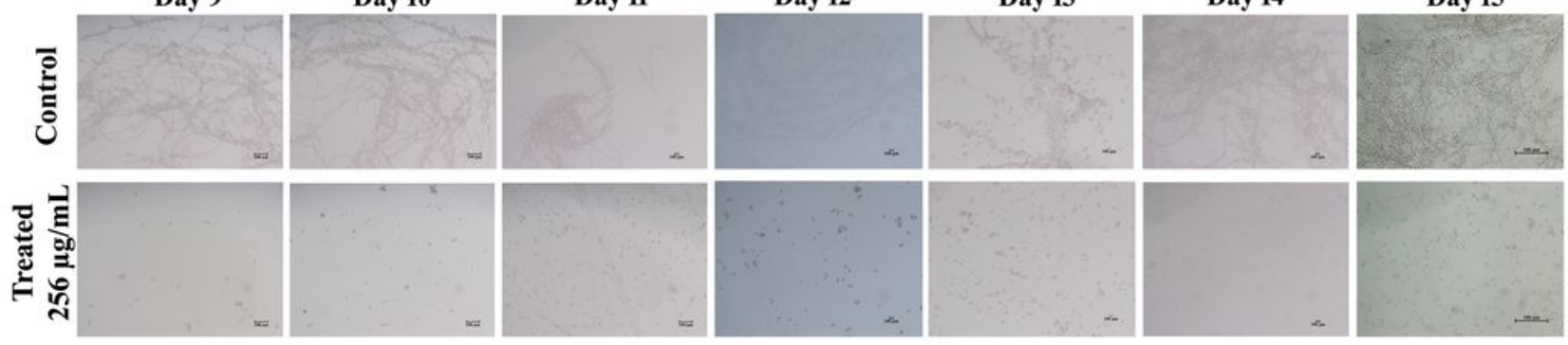

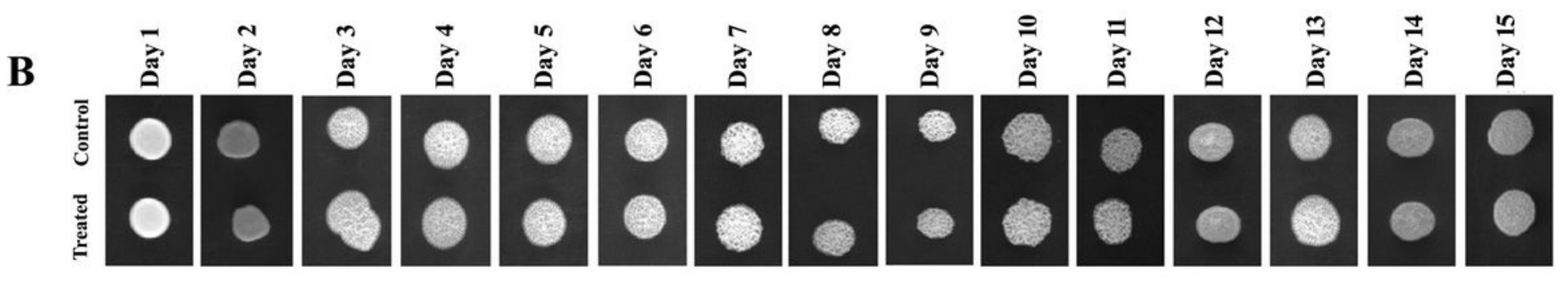

Figure 14

Serial passage experiment by repeated exposure of catechol to $C$. albicans cells on both YEPD and spider liquid media. (A) Light graphs were displayed the potential of catechol in inhibiting the yeast to hyphae transition even after serial 15 passages. (Magnification - $200 \times$, Scale bar $-100 \mu \mathrm{m}$ ). (B) Spot assay revealed non-impairment of $\mathrm{C}$. albicans metabolic growth in presence and absence of catechol even after 15 serial passages. 
A

B
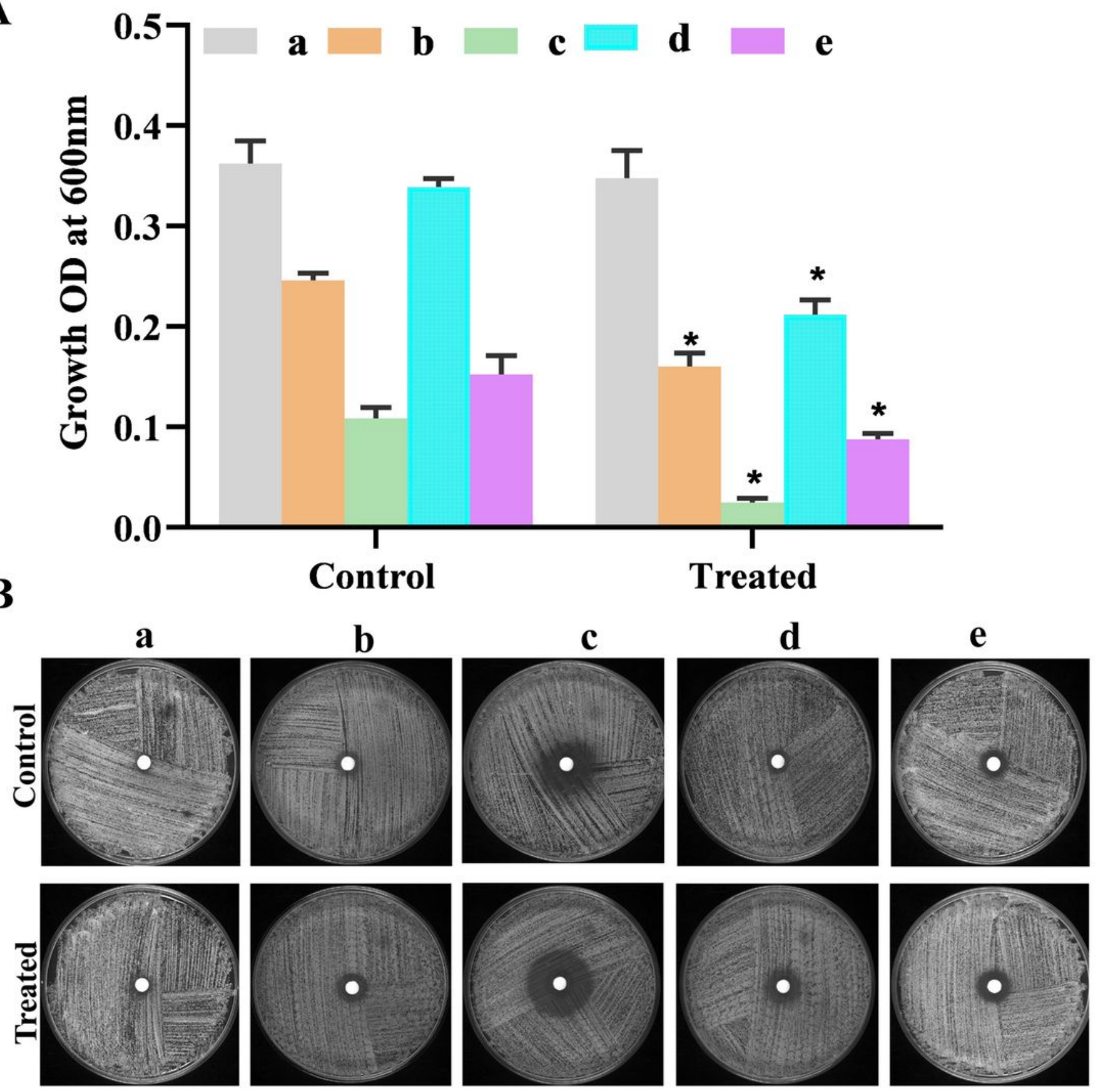

Figure 15

(A) Antifungal susceptibility testing of catechol pretreated (3 h) C. albicans cells after $24 \mathrm{~h}$ incubation using broth micro dilution assay. (B) Antifungal susceptibility testing of catechol pretreated C. albicans cells to conventional antifungals using disc diffusion method.(a) Flucanozole (b) micanozole (c) ketoconazole (d) nystain and (e) amphotericin-B. Error bars indicates the mean values of three experimental duplicates. The "*

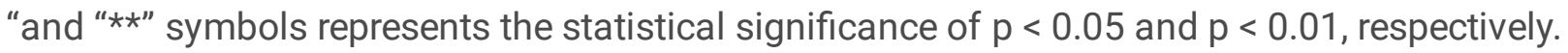




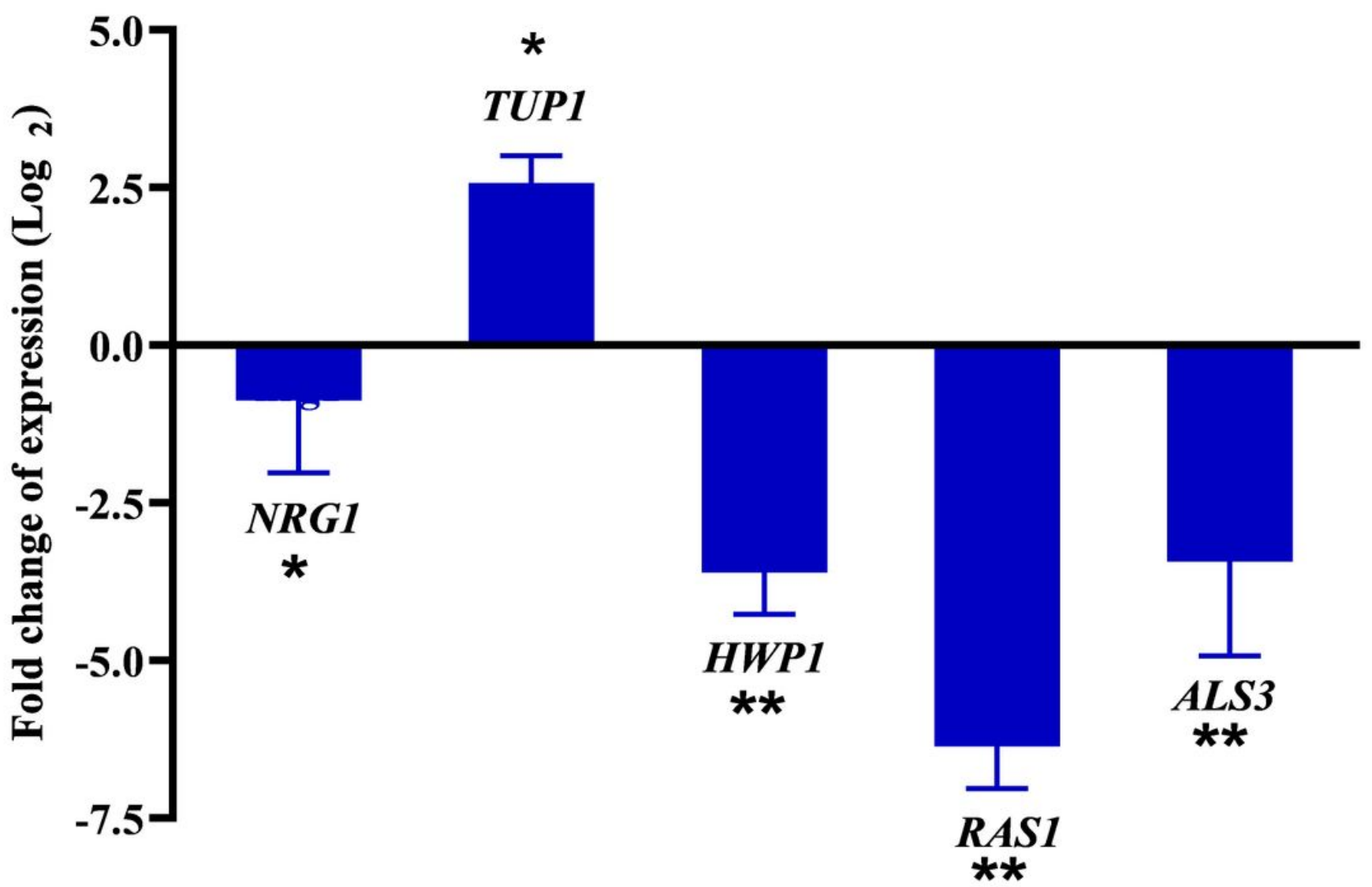

Figure 16

Expression profiles of used candidate genes at transcriptomic level in the presence and absence of catechol.

The genes responsible for adhesion, filamentous growth and biofilm formation such as HWP1, RAS1, and ALS3 were significantly downregulated in catechol treated cells. TUP1, a negative transcription regulator of filamentation was upregulated. The relative gene expression was calculated by $2-\Delta \Delta C T$ method using $C$. albicans ITS region as housekeeping internal control gene. Error bars indicates the mean values of three experimental duplicates. The " "and " $\star \star$ " symbols represents the statistical significance of $p<0.05$ and $p<0.01$, respectively. 


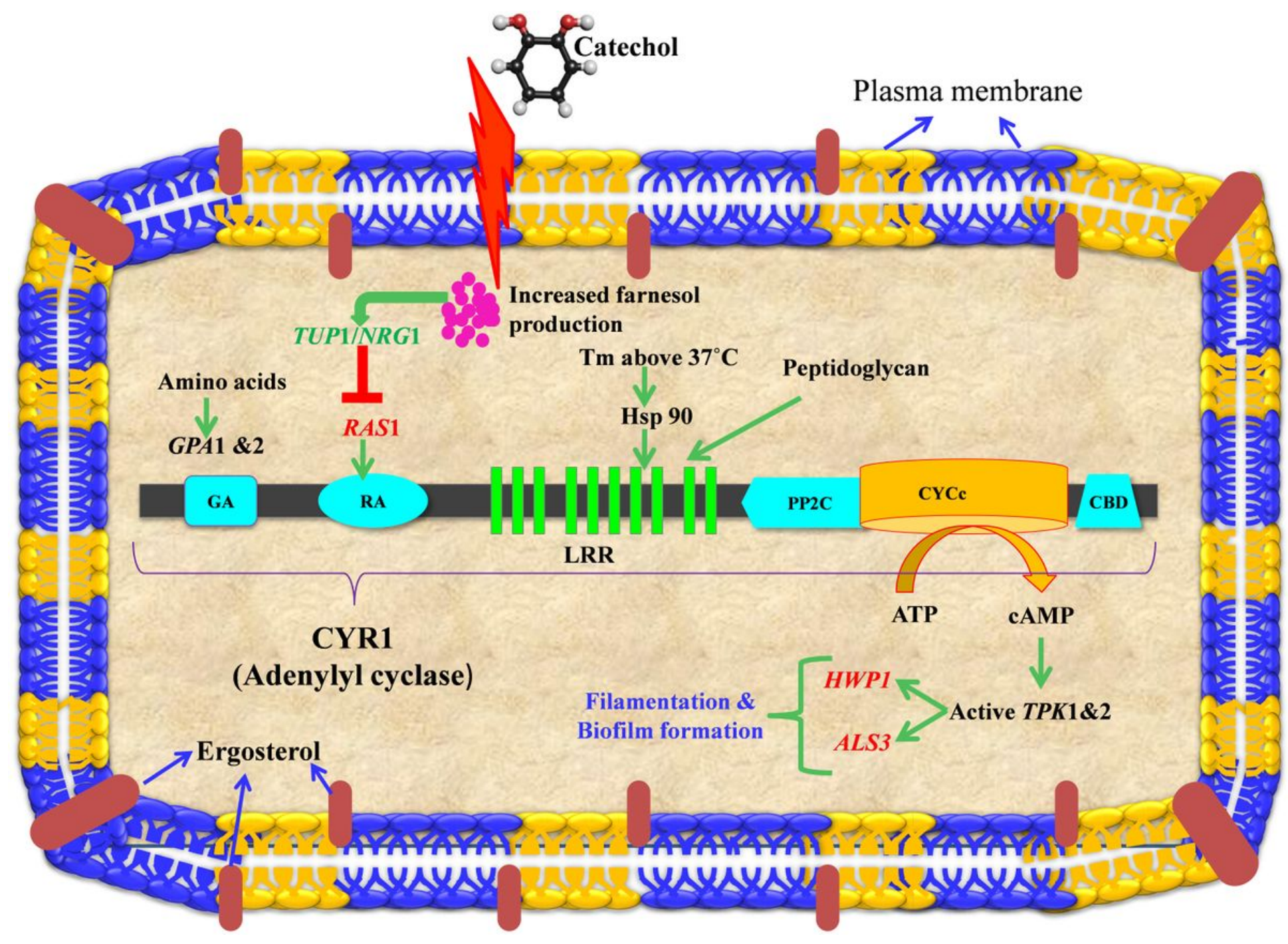

Figure 17

Schematic illustration of differential expression of genes involved in C. albicans Ras-cAMP-PKA pathway during catechol exposure. Unregulated and down regulated genes were indicated using red and green colored arrow, respectively. 